\title{
DYNAMICAL SYSTEMS IN PERTURBATIVE SCALAR FIELD COSMOLOGY
}

\author{
Artur Alho, ${ }^{1 *}$, Claes Uggla,${ }^{2 \dagger}$ And John Wainwright ${ }^{3 \ddagger}$ \\ ${ }^{1}$ Center for Mathematical Analysis, Geometry and Dynamical Systems, \\ Instituto Superior Técnico, Universidade de Lisboa, \\ Av. Rovisco Pais, 1049-001 Lisboa, Portugal. \\ ${ }^{2}$ Department of Physics, Karlstad University, \\ S-65188 Karlstad, Sweden. \\ ${ }^{3}$ Department of Applied Mathematics, University of Waterloo, \\ Waterloo, ON, N2L 3G1, Canada.
}

\begin{abstract}
We derive a new regular dynamical system on a 3-dimensional compact state space describing linear scalar perturbations of spatially flat RobertsonWalker geometries for relativistic models with a minimally coupled scalar field with an exponential potential. This enables us to construct the global solution space, illustrated with figures, where known solutions are shown to reside on special invariant sets. We also use our dynamical systems approach to obtain new results about the comoving and uniform density curvature perturbations. Finally we show how to extend our approach to more general scalar field potentials. This leads to state spaces where the state space of the models with an exponential potential appears as invariant boundary sets, thereby illustrating their role as building blocks in a hierarchy of increasingly complex cosmological models.
\end{abstract}

\section{Introduction}

Inflation, quintessence - scalar fields appear prominently in standard cosmology, where the universe on large scales is described by a perturbed Robertson-Walker (RW) background. But a lack of consensus, e.g. about interpretation of observations, first principles, and issues such as fine tuning of initial data, has resulted in a plethora of proposed models and scalar field potentials, often connected with various heuristic physical and mathematical considerations. This suggests that there might be some value in a systematic approach using dynamical systems to analyze the evolution

*Electronic address:artur.alho@tecnico.ulisboa.pt

${ }^{\dagger}$ Electronic address:claes.uggla@kau.se

${ }^{\ddagger}$ Electronic address:jwainwri@uwaterloo.ca 
of cosmological models on perturbed RW backgrounds, especially those involving scalar fields. With this goal in mind, we have embarked on a research program using dynamical systems, where this paper is the second in a series of papers (the first, Alho et al (2019) [1, treated scalar and tensor perturbations of $\Lambda$ CDM models).

A dynamical system consists of a system of autonomous nonlinear first order ordinary differential equations (ODEs). In the applications in cosmology that we have in mind the state space $\mathcal{S}$ has a product structure

$$
\mathcal{S}=\mathcal{B} \times \mathcal{P}
$$

where $\mathcal{B}$ is the background state space, which describes the dynamics of a RW background, and $\mathcal{P}$ is the perturbation state space, which contains Fourier decomposed gauge invariant variables that describe linear perturbations. In this paper we, for brevity, neglect vector and tensor perturbation modes. The system of differential equations has one subset of equations that acts on $\mathcal{B}$ while the remaining equations act on $\mathcal{P}$ with coefficients from $\mathcal{B}$. In this way the dynamics in the background determine the dynamics of the perturbations. A key step in our approach is to choose variables that lead to regular equations on a bounded state space. This enables one to give a global description of the dynamics, in particular, the behaviour at early and late times and the evolution at intermediate stages that may be of physical interest. In addition the differential equations are well-suited for performing systematic numerical simulations.

In Alho et al (2019) [1] the background state space describes the $\Lambda$ CDM model, which represents the simplest situation since both $\mathcal{B}$ and $\mathcal{P}$ are one-dimensional sets, both for scalar and tensor perturbations. In the present paper the background state space $\mathcal{B}$ describes a spatially flat RW model with a minimally coupled scalar field. In order to show how to incorporate such a source within our new framework we have focused on the simplest potential, the exponential potential, $V(\varphi)=V_{0} \exp (-\sqrt{6} \lambda \varphi)$, where $V_{0}$ and $\lambda$ are constants. In this case the background space $\mathcal{B}$ has dimension 2 and the perturbation space $\mathcal{P}$ has dimension 1 , yielding a bounded state space $\mathcal{B} \times \mathcal{P}$ of dimension 3 . Using our approach we are able to give a complete picture of the dynamics, showing all ways in which a model can evolve from early to late times, illustrated by figures with representative orbits. The value of the parameter $\lambda$ in the potential determines two disparate families of models, those with future acceleration and deceleration, respectively.

As regards earlier analytical work, the perturbation equations have been solved explicitly in terms of Bessel functions, by imposing the restriction that the scale factor $a$ has a power law dependence on conformal time, which corresponds to a constant deceleration parameter 11 We show that these well known solutions are described by orbits (solution trajectories) on a two dimensional invariant subset of the three dimensional state space $\mathcal{B} \times \mathcal{P}$ that contains the future attractor of the whole state space. These special solutions thus play a central role in the dynamics

\footnotetext{
${ }^{1}$ See for example Lyth and Stewart (1992) [2], equations (21) and (22), Durrer (2008) 3], page 113, and Weinberg (2008) 4] pages 480-482. These references describe the primary application of the exponential scalar field potential in inflationary cosmology, namely, determining the power spectrum for perturbations in power law inflation.
} 
of scalar fields with exponential potential, but do not describe the full range of dynamic possibilities.

Our dynamical systems treatment also sheds light on the comoving and uniform density curvature perturbations. In particular, it provides a new context to recent discussions about the so-called ultra slow-roll inflation and conserved quantities, see e.g. [5, 6, 7, 8, 9, 10, 11].

The outline of the paper is as follows. In the next section we present the background Einstein and (non-linear) Klein-Gordon (KG) equations for the spatially flat RW background with a matter content given by a minimally coupled scalar field, and we give the perturbed KG equation specialized to the uniform (flat) curvature gauge. In Section 3 we formulate the background equations and the linearly perturbed KG equation as a three-dimensional dynamical system with regular differential equations on a compact state space $\mathcal{B} \times \mathcal{P}$. This is followed by a dynamical systems analysis in Section 4, where figures with representative orbits globally illustrate the entire state space. In addition we present analytic asymptotic descriptions of the orbits at late and early times. In Section 5 we review the well known explicit solutions and relate them to our dynamical systems framework. In Section 6 we derive new analytic asymptotic results for the comoving and uniform density curvature perturbations and relate them to recent research in the case of ultra slow-roll inflation. Section 7 shows how our dynamical systems approach can be extended to a hierarchy of increasingly complex models.

\section{Field equations for a minimally coupled scalar field}

\subsection{The background equations}

We consider a spatially flat RW background with a metric written as

$$
d s^{2}=-d t^{2}+a^{2} \gamma_{i j} d x^{i} d x^{j}=a^{2}\left(-d \eta^{2}+\gamma_{i j} d x^{i} d x^{j}\right)=-H^{-2} d N^{2}+a^{2} \gamma_{i j} d x^{i} d x^{j},
$$

where $a$ is the background scale factor, $H$ the Hubble variable, and $\gamma_{i j}$ is the flat spatial 3-metric, which in Cartesian coordinates is given by $\delta_{i j}$. The different time coordinates above are the clock time $t$, the conformal time $\eta$, and the $e$-fold time

$$
N=\ln \left(a / a_{0}\right)
$$

where $N$ describes the number of background $e$-foldings with respect to some reference epoch at which $a=a_{0}$ and hence $N=0$.

The background equations that govern the matter distribution in a flat RW universe are the Friedman equation and the energy conservation equation

$$
\rho=3 H^{2}, \quad \dot{\rho}=-3 H(\rho+p) .
$$

where overdot denotes derivative with respect to $t$. 
The energy density $\rho$ and pressure $p$ for a minimally coupled scalar field $\varphi$ with a non-negative potential, $V=V(\varphi)$ are given by $\left.\right|^{2}$

$$
\rho=\frac{1}{2} \dot{\varphi}^{2}+V(\varphi), \quad \rho+p=\dot{\varphi}^{2},
$$

Making the obvious substitutions of equations (5) into (4) we obtain ${ }^{3}$

$$
\ddot{\varphi}+3 H \dot{\varphi}+V_{, \varphi}=0, \quad 3 H^{2}=\frac{1}{2} \dot{\varphi}^{2}+V(\varphi),
$$

where ${ }_{, \varphi}$ denotes $\frac{d}{d \varphi}$. Given a potential $V(\varphi)$ these equations determine $\varphi(t)$ and $H(t)$ when $\varphi$ and $\dot{\varphi}$ are specified at an initial time. The equation $\dot{a}=a H$ then determines the scale factor $a$ as a quadrature.

Before continuing we digress to introduce the deceleration parameter $q$ which is useful in describing the dynamics:

$$
\dot{H}=-(1+q) H^{2} .
$$

It follows from (4) and (5) that

$$
1+q=\frac{1}{2} \frac{\dot{\varphi}^{2}}{H^{2}}
$$

We find it convenient to use the $e$-fold time $N$ instead of the clock time $t$ as our starting point and to introduce the following quantities: ${ }^{4}$

$$
\Sigma_{\varphi}=\frac{1}{\sqrt{6}} \varphi^{\prime}, \quad \Omega_{V}=\frac{V}{3 H^{2}}, \quad \lambda=-\frac{1}{\sqrt{6}} \frac{V_{, \varphi}}{V},
$$

where $\mathrm{a}^{\prime}$ denotes the $e$-fold time derivative $\frac{d}{d N}=H^{-1} \frac{d}{d t}$. Equations (7) and (8) assume the form

$$
H^{\prime}=-(1+q) H, \quad 1+q=3 \Sigma_{\varphi}^{2} .
$$

The definitions (9) have the effect of eliminating the explicit appearance of $H$ in the governing equations (6). The second equation becomes

$$
\Sigma_{\varphi}^{2}+\Omega_{V}=1
$$

while the second order ODE is replaced by two coupled first order ODEs:

$$
\begin{aligned}
\varphi^{\prime} & =\sqrt{6} \Sigma_{\varphi}, \\
\Sigma_{\varphi}^{\prime} & =3\left(1-\Sigma_{\varphi}^{2}\right)\left(\lambda-\Sigma_{\varphi}\right) .
\end{aligned}
$$

\footnotetext{
${ }^{2}$ See for example Liddle and Lyth (2000) [12, equations (3.3) and (3.4).

${ }^{3}$ See for example Liddle and Lyth (2000) [12], equations (3.5) and (3.6).

${ }^{4}$ The factors of $\sqrt{6}$ are included in the definitions $\sqrt{9}$ in order to simplify future algebra. The reason for using the notation $\Sigma$ for the kernel is because this variable plays a similar role as Hubble-normalized shear, which is typically denoted with the kernel $\Sigma$, see e.g. [13].
}

${ }^{5}$ As a first step divide both equations by $H^{2}$ and note that

$$
\frac{d}{d t}=H \frac{d}{d N}, \quad \frac{d^{2}}{d t^{2}}=H^{2}\left(\frac{d^{2}}{d N^{2}}-(1+q) \frac{d}{d N}\right) .
$$

Use (10) to eliminate $1+q$, and use the definition of $\lambda$ in (9) and (11) to eliminate $V_{, \varphi}$ in (6). 
In general the scalar $\lambda$, defined by $(9)$, is a function of $\varphi$ but in the case of an exponential potential $\lambda$ is constant. We use this constant value to define $V$ according td 6

$$
V=V_{0} \exp (-\sqrt{6} \lambda \varphi), \quad V_{0}>0 .
$$

Since $\lambda$ is now a constant (12a) decouples from $12 \mathrm{~b})$, and can be used to determine $\varphi$ by quadrature once $(12 \mathrm{~b}$ ) has been solved. Thus $12 \mathrm{~b}$ contains the essential dynamical information for the background scalar field in the case of an exponential potential. Since $V$ has been defined to be positive, it follows from $(9)$ that $\Omega_{V}$ is positive and hence from (11) that $\Sigma_{\varphi}$ is bounded: $-1<\Sigma_{\varphi}<1$. However, since the right hand side of (12b) is continuously differentiable and zero when $\Sigma_{\varphi}= \pm 1$ it is possible and desirable to extend the range of $\Sigma_{\varphi}$ to include the invariant boundary values $\Sigma_{\varphi}= \pm 1$ :

$$
-1 \leq \Sigma_{\varphi} \leq 1
$$

Equation (10) now restricts the deceleration parameter:

$$
-1 \leq q \leq 2
$$

where $q=-1$ if $\Sigma_{\varphi}=0$ and $q=2$ if $\Sigma_{\varphi}= \pm 1$.

\subsection{The perturbed Klein-Gordon equation}

To describe the linear perturbations of the scalar field we use the perturbed KG equation, which has a particularly simple form if one uses as dependent variable the linear perturbation of the scalar field in the uniform (flat) curvature gauge.$^{7}$ which we denote by $\varphi_{\mathrm{c}}$. An advantage of using the uniform curvature gauge is that the metric perturbations can be eliminated from the KG equation using the Einstein equations, leaving a closed equation which can be written as follows (see, e.g., Uggla and Wainwright (2019) [16], section 3, Appendix A.3 and in particular equation $(\mathrm{A} .21))$ :

$$
\partial_{N}^{2} \varphi_{\mathrm{c}}+\frac{V}{H^{2}} \partial_{N} \varphi_{\mathrm{c}}+\frac{\left(V_{, \varphi \varphi}+2 \varphi^{\prime} V_{, \varphi}+\left(\varphi^{\prime}\right)^{2} V\right)}{H^{2}} \varphi_{\mathrm{c}}-\mathcal{H}^{-2} \mathbf{D}^{2} \varphi_{\mathrm{c}}=0
$$

where $\varphi$ is the background scalar field,

$$
\mathcal{H}=a H
$$

and $\mathbf{D}^{2}$ is the background spatial Laplacian associated with $\gamma_{i j}$. For an exponential potential (13) the KG equation assumes the form

$$
\partial_{N}^{2} \varphi_{\mathrm{c}}+3\left(1-\Sigma_{\varphi}^{2}\right) \partial_{N} \varphi_{\mathrm{c}}+18\left(1-\Sigma_{\varphi}^{2}\right)\left(\lambda-\Sigma_{\varphi}\right)^{2} \varphi_{\mathrm{c}}-\mathcal{H}^{-2} \mathbf{D}^{2} \varphi_{\mathrm{c}}=0
$$

in the notation of $(9)$.

\footnotetext{
${ }^{6}$ This labelling of $V$ is consistent with the definition $(9)$ of $\lambda$.

${ }^{7}$ Once one chooses the uniform curvature gauge or the comoving gauge as we do in section 6 . there is no remaining gauge freedom. See for example [14] or [15] section 3 .
} 


\section{Derivation of the dynamical system}

To obtain a dynamical system, i.e., an autonomous closed system of first order ODEs, that describes the evolution of the perturbations, we first introduce Cartesian spatial coordinates and make a spatial Fourier decomposition of the perturbation variables. This results in

$$
\mathcal{H}^{-2} \mathbf{D}^{2} \rightarrow-k^{2} \mathcal{H}^{-2}
$$

thereby transforming $(18)$ to

$$
\varphi_{\mathrm{c}}^{\prime \prime}+3\left(1-\Sigma_{\varphi}^{2}\right) \varphi_{\mathrm{c}}^{\prime}+18\left(1-\Sigma_{\varphi}^{2}\right)\left(\lambda-\Sigma_{\varphi}\right)^{2} \varphi_{\mathrm{c}}+k^{2} \mathcal{H}^{-2} \varphi_{\mathrm{c}}=0,
$$

where $k$ is the wave number. The Fourier coefficients of $\varphi_{\mathrm{c}}$ are labelled by an index $k,\left(\varphi_{\mathrm{c}}\right)_{k}$, but for brevity we will drop the index $k$ when there is no danger of confusion. At this stage we have to take into account that the Fourier coefficients $\varphi_{\mathrm{c}}$ are complex functions ${ }^{8}$ Since we want to obtain a dynamical system in terms of real variables we write $\varphi_{\mathrm{c}}=f_{1}+i f_{2}$, where $f_{1}$ and $f_{2}$ are real functions, labelled by the wave number $k$, which describe the general solution for a given $k$. Since 200 is a linear differential equation with real coefficients it follows that $f_{1}, f_{2}$ satisfy this differential equation which we rewrite as

$$
f^{\prime \prime}+3\left(1-\Sigma_{\varphi}^{2}\right) f^{\prime}+18\left(1-\Sigma_{\varphi}^{2}\right)\left(\lambda-\Sigma_{\varphi}\right)^{2} f+k^{2} \mathcal{H}^{-2} f=0,
$$

where $f$ stands for $f_{1}$ or $f_{2}$. We now consider $f$ and $f^{\prime}$, with subscript 1 or 2 , as independent variables and represent them using polar coordinates $f_{i}=r_{i} \cos \theta_{i}$, $f_{i}^{\prime}=r_{i} \sin \theta_{i}$, for $i=1,2$, where $\theta$ has period $2 \pi$. Note that the variables $f$ and $\theta$ are related by the equation

$$
f^{\prime}=f \tan \theta
$$

for both values of the index $i$. Differentiating this equation to obtain $f^{\prime \prime}$ converts equation (21) into the following first order differential equation for $\theta$ :

$$
\theta^{\prime}=-\sin ^{2} \theta-3\left(1-\Sigma_{\varphi}^{2}\right) \sin \theta \cos \theta-\left[18\left(1-\Sigma_{\varphi}^{2}\right)\left(\lambda-\Sigma_{\varphi}\right)^{2}+k^{2} \mathcal{H}^{-2}\right] \cos ^{2} \theta,
$$

where $\theta$ stands for $\theta_{1}$ or $\theta_{2}$. The angular variable $\theta$ will be useful for describing the global structure of the state space. For doing local calculations, however, it is more convenient to use the variable $y$ defined by

$$
y=\frac{f^{\prime}}{f}=\tan \theta,
$$

where $y$ stands for $y_{1}$ or $y_{2}$. Since $y^{\prime}=\left(\sec ^{2} \theta\right) \theta^{\prime}$, equation (23) leads to the Riccati equation

$$
y^{\prime}=-\left[y^{2}+3\left(1-\Sigma_{\varphi}^{2}\right) y+18\left(1-\Sigma_{\varphi}^{2}\right)\left(\lambda-\Sigma_{\varphi}\right)^{2}\right]-k^{2} \mathcal{H}^{-2} .
$$

\footnotetext{
${ }^{8}$ The physical perturbation $\varphi_{\mathrm{c}}$ of the scalar field is assumed to be real. Note also that the Fourier coefficients $\varphi_{\mathrm{c}, \mathbf{k}}$ and $\varphi_{\mathrm{c},-\mathbf{k}}$, where $\mathbf{k}$ is the wave vector, obey the same equation since the wave vector $\mathbf{k}$ only enters the problem via the wave number as $k^{2}$, which is why we have denoted the Fourier coefficients by $k$. For a discussion on Fourier decompositions for cosmological perturbations, see [1] and references therein.
} 
In summary, a complex solution $\varphi_{\mathrm{c}}(N)=f_{1}(N)+i f_{2}(N)$ of the KG equation (20), which is a second order linear differential equation, is described by two real solutions $y_{1}(N), y_{2}(N)$ of the first order non-linear differential equation (25). The functions $y_{i}$ determine the functions $f_{i}$ by quadrature using (24), which yields

$$
f_{i}(N)=f_{i}(0) \exp \left[\int_{0}^{N} y_{i}(\tilde{N}) d \tilde{N}\right]
$$

where the initial values $f_{i}(0)$ are determined by the initial value of $\varphi_{\mathrm{c}}$ at $N=0$.

Equations (23) and (25) are first order non-autonomous ODEs with time dependent coefficients that depend on the background solutions for $\Sigma_{\varphi}$ and $\mathcal{H}^{-2}$. The wave number $k$ only appears in the equations as a parameter in the dimensionless combination $k^{2} \mathcal{H}^{-2}$. To obtain a dynamical system, i.e., an autonomous closed system of first order ODEs, we need to augment the ODE (25) for $y$ (or (23) for $\theta$ ) by including the ODE (12b) for $\Sigma_{\varphi}$ and an ODE for $k^{2} \mathcal{H}^{-2}$ when $k \neq 0$. We therefore introduce a new variable $Z$ (referred to as the physical wave number in Hubble units in [7]):

$$
Z=k^{2} \mathcal{H}^{-2}
$$

which satisfies the differential equation

$$
Z^{\prime}=2 q Z=2\left(3 \Sigma_{\varphi}^{2}-1\right) Z
$$

on account of (10), (17) and $a^{\prime}=a$.

For the state space described by $\left(\Sigma_{\varphi}, Z, y\right)$ we thereby obtain the following dynamical system:

$$
\begin{aligned}
\Sigma_{\varphi}^{\prime} & =3\left(1-\Sigma_{\varphi}^{2}\right)\left(\lambda-\Sigma_{\varphi}\right), \\
Z^{\prime} & =2\left(3 \Sigma_{\varphi}^{2}-1\right) Z, \\
y^{\prime} & =-\left[y^{2}+3\left(1-\Sigma_{\varphi}^{2}\right) y+18\left(1-\Sigma_{\varphi}^{2}\right)\left(\lambda-\Sigma_{\varphi}\right)^{2}\right]-Z,
\end{aligned}
$$

while for the state space described by $\left(\Sigma_{\varphi}, Z, \theta\right)$, where we recall that $y=\tan \theta$, the above equation for $y$ is replaced with

$$
\theta^{\prime}=-\left[\sin ^{2} \theta+3\left(1-\Sigma_{\varphi}^{2}\right) \sin \theta \cos \theta+18\left(1-\Sigma_{\varphi}^{2}\right)\left(\lambda-\Sigma_{\varphi}\right)^{2} \cos ^{2} \theta\right]-Z \cos ^{2} \theta .
$$

The dynamical systems for the state spaces $\left(\Sigma_{\varphi}, Z, y\right)$ and $\left(\Sigma_{\varphi}, Z, \theta\right)$ are regular in the sense that the expressions on the right hand sides are differentiable functions of the state space variables. Note also that the two ODEs $(29 \mathrm{a})$ and $(29 \mathrm{~b})$, which describe the background state space $\left(\Sigma_{\varphi}, Z\right)$, are uncoupled since $Z$ does not appear in 29a). Thus one can solve 29a for $\Sigma_{\varphi}$ and then express $Z$ as a quadrature using (29b).

Before continuing we comment on the interpretation of the variable $Z=k^{2} \mathcal{H}^{-2}$. Perturbations that satisfy $k^{2} \mathcal{H}^{-2} \ll 1$ are called long wavelength or super-horizon, while those that satisfy $k^{2} \mathcal{H}^{-2} \gg 1$ are said to be short wavelength. Long wavelength perturbations are usually studied by choosing the idealized limiting value $k=0$ ?

\footnotetext{
${ }^{9}$ The long wavelength case $(k=0)$ can be solved explicitly for any scalar field potential, even up to second order, as shown in [16.
} 
which corresponds to $Z=0$. On the other hand, short wavelength perturbations correspond to $Z \rightarrow \infty$. We also note that in choosing $Z=k^{2} \mathcal{H}^{-2}$ as a dynamical variable we have in a sense 'hidden' the wave number $k$ when formulating the dynamical system. However, if we choose the reference time $t_{0}$ (i.e., when $N=0$ ) to be the time for setting initial data in the state space $\left(\Sigma_{\varphi}, Z, y\right)$ (or $\left.\left(\Sigma_{\varphi}, Z, \theta\right)\right)$, then different choices of $Z_{0}=k^{2} \mathcal{H}_{0}^{-2}$ for a given $\mathcal{H}_{0}$ yield solutions with different wave number $k$.

A drawback of the system (29) is that the variable $Z$ can become unbounded for some models ${ }^{10}$ To deal with this situation we introduce the bounded variable

$$
\bar{Z}=\frac{Z}{1+Z}=\frac{k^{2}}{k^{2}+\mathcal{H}^{2}}, \quad Z=\frac{\bar{Z}}{1-\bar{Z}},
$$

which due to (28) obeys the equation

$$
\bar{Z}^{\prime}=2\left(3 \Sigma_{\varphi}^{2}-1\right) \bar{Z}(1-\bar{Z}),
$$

where the long (short) wavelength limit corresponds to $\bar{Z}=0(\bar{Z}=1)$. This change of variable, however, leads to the appearance of the term $\bar{Z} /(1-\bar{Z})$, which is unbounded when $\bar{Z}=1$, in the equations for $y$ and $\theta$. To regularize the dynamical system we choose a new time variable $\bar{N}$ according to

$$
\frac{d \bar{N}}{d N}=\frac{1}{1-\bar{Z}}=1+Z \text {. }
$$

This results in the following regular dynamical system:

$$
\begin{aligned}
& \frac{d \Sigma_{\varphi}}{d \bar{N}}=3\left(1-\Sigma_{\varphi}^{2}\right)\left(\lambda-\Sigma_{\varphi}\right)(1-\bar{Z}), \\
& \frac{d \bar{Z}}{d \bar{N}}=2\left(3 \Sigma_{\varphi}^{2}-1\right) \bar{Z}(1-\bar{Z})^{2}, \\
& \frac{d y}{d \bar{N}}=-\left[y^{2}+3\left(1-\Sigma_{\varphi}^{2}\right) y+18\left(1-\Sigma_{\varphi}^{2}\right)\left(\lambda-\Sigma_{\varphi}\right)^{2}\right](1-\bar{Z})-\bar{Z},
\end{aligned}
$$

on the state space $\left(\Sigma_{\varphi}, \bar{Z}, y\right)$ defined by

$$
-1 \leq \Sigma_{\varphi} \leq 1, \quad 0 \leq \bar{Z} \leq 1, \quad-\infty<y<\infty .
$$

If we replace $y$ by the angular variable $\theta$ of period $2 \pi$, equation (34c) is replaced by

$$
\begin{aligned}
\frac{d \theta}{d \bar{N}}= & -\left[\sin ^{2} \theta+3\left(1-\Sigma_{\varphi}^{2}\right) \sin \theta \cos \theta+18\left(1-\Sigma_{\varphi}^{2}\right)\left(\lambda-\Sigma_{\varphi}\right)^{2} \cos ^{2} \theta\right](1-\bar{Z}) \\
& -\bar{Z} \cos ^{2} \theta,
\end{aligned}
$$

which results in a state space that is bounded (and compact) ${ }^{11}$

\footnotetext{
${ }^{10}$ The variable $Z$ becomes unbounded when $k \neq 0$ and $\mathcal{H} \rightarrow 0$. However, since $q$ is bounded for the present models $\mathcal{H} \rightarrow 0$ only asymptotically toward the past or the future, depending on the asymptotic signs of $q$.

${ }^{11}$ At this stage the reader might ask why we used the variable $\varphi_{\mathrm{c}}$ and the KG equation as our starting point and not metric perturbation variables and the Einstein field equations in, e.g., the uniform curvature gauge. The reason is that in the latter case $\Sigma_{\varphi}$ occurs in the denominator, which leads to that the perturbed Einstein field equations break down when $\Sigma_{\varphi}=0$.
} 


\section{Analysis of the dynamical system}

In this section we use the dynamical system that is described by the differential equations (34) and (36) on the state space $\mathcal{S}=\mathcal{B} \times \mathcal{P}$ to give a complete description of the dynamics of a perturbed scalar field with exponential potential. The dynamical system depends on the parameter $\lambda$ which we assume satisfies $0 \leq \lambda<1$, since these are the values of primary physical interest ${ }^{12}$

\subsection{Invariant sets and fixed points in $\mathcal{B} \times \mathcal{P}$}

The state space $\mathcal{S}=\mathcal{B} \times \mathcal{P}$ is a three-dimensional product space where $\mathcal{B}$ is the background state space, with coordinates $\Sigma_{\varphi}$ and $\bar{Z}$, subject to

$$
-1 \leq \Sigma_{\varphi} \leq 1, \quad 0 \leq \bar{Z} \leq 1,
$$

while $\mathcal{P}$ is the perturbation state space, which is a circle parameterized by the angular coordinate $\theta$ of period $2 \pi$. We can visualize $\mathcal{S}$ by rotating the rectangle (37) through $2 \pi$ radians about a line $\Sigma_{\varphi}=b<-1$ to form a solid finite cylinder with a cylindrical hole along its axis. The surfaces of constant $\Sigma_{\varphi}$ are coaxial cylinders whose common axis defines the $\bar{Z}$-axis with $0 \leq \bar{Z} \leq 1$. The cylinders $\Sigma_{\varphi}=1$ and $\Sigma_{\varphi}=-1$ form the outer and inner boundaries of the solid cylinder, respectively, and are invariant sets of the dynamical system, as follows from equation (34a). The planes $\bar{Z}=0$ and $\bar{Z}=1$ form the bottom and top of the solid cylinder, respectively, and are also invariant sets as follows from equation (34b). In this representation the state space $\mathcal{B} \times \mathcal{P}$ is a compact subset of $\mathbb{R}^{3}$. On the other hand if we use the perturbation variable $y=\tan \theta$ then the state space $\mathcal{B} \times \mathcal{P}$ is the infinite slab in $\mathbb{R}^{3}$ defined by the inequalities (35) ${ }^{13}$

The invariant sets $\Sigma_{\varphi}=1$ and $\Sigma_{\varphi}=-1$ describe the limiting case of a massless scalar field model $(V=0$, see equations $(9)$ and $(12))$. We will therefore refer to the invariant sets $\Sigma_{\varphi}= \pm 1$ as the two components of the massless scalar field boundary. Because of the physical interpretation of $\bar{Z}$ (see section 3 ) we refer to the invariant set $\bar{Z}=0$ as the long wavelength boundary, and $\bar{Z}=1$ as the short wavelength boundary. In addition to the above invariant boundary sets there is an important invariant set given by the interior cylinder with $\Sigma_{\varphi}=\lambda$.

We obtain the fixed points of the dynamical system by equating the right hand sides of (34) to zero. It follows that if $0 \leq \lambda<1$ and $\lambda \neq 1 / \sqrt{3}$ then all the fixed points of the dynamical system lie in the long and short wavelength boundary sets

\footnotetext{
${ }^{12}$ Firstly, there is no restriction in assuming that $\lambda$ is non-negative, since the field equations are invariant under the transformation $\left(\varphi, \Sigma_{\varphi}\right) \rightarrow-\left(\varphi, \Sigma_{\varphi}\right)$ and $\lambda \rightarrow-\lambda$. Secondly, the fixed point $\Sigma_{\varphi}=\lambda$ is the future attractor for the $\Sigma_{\varphi}$ background state space. At this fixed point the deceleration parameter $q=3 \lambda^{2}-1$ and is thus constant. The range $0 \leq \lambda \leq 1$ corresponds to the range $-1 \leq q \leq 1$ for $q$. This range of $q$ also describes a space-time with a perfect fluid with a linear equation of state $p=w \rho$, with $w$ in the range $-1 \leq w \leq 1$. Thus $\lambda=0$ corresponds to a cosmological constant while the bifurcation value $\lambda=1$ corresponds to a stiff fluid with speed of sound equal to that of light. On the other hand $\lambda>1$ yields an equation of state with superluminal speed.

${ }^{13}$ Since $\tan (\theta+\pi)=\tan \theta$, the mapping $y=\tan \theta$ is two-to-one and therefore when $\theta$ makes one revolution $(0 \rightarrow 2 \pi) y$ has to be traversed twice $-\infty \rightarrow+\infty$.
} 
$\bar{Z}=0$ and $\bar{Z}=1$. On the $\bar{Z}=0$ boundary they either satisfy $\Sigma_{\varphi}=\lambda$ or $\Sigma_{\varphi}^{2}=1$. There are two hyperbolic fixed points with $\Sigma_{\varphi}=\lambda$ which we denote by $\mathrm{A}_{\lambda}$ and $\mathrm{S}_{\lambda}$ :

$$
\begin{array}{ll}
\mathrm{A}_{\lambda}: & \Sigma_{\varphi}=\lambda, \quad \bar{Z}=0, \quad y=0, \\
\mathrm{~S}_{\lambda}: & \Sigma_{\varphi}=\lambda, \quad \bar{Z}=0, \quad y=-3\left(1-\lambda^{2}\right) .
\end{array}
$$

There are also two non-hyperbolic fixed points denoted by $\mathrm{M}_{ \pm}$, given by

$$
\mathrm{M}_{ \pm}: \quad \Sigma_{\varphi}= \pm 1, \quad \bar{Z}=0, \quad y=0 .
$$

However, when we draw pictures of the global state space we will use $\theta$ as the perturbation variable, with $\tan \theta=y$. Then $y=0$ corresponds to $\theta=n \pi, n=0,1$, where $y=-3\left(1-\lambda^{2}\right)$ corresponds to $\theta=\chi+n \pi, n=0,1$, where $\chi=-\arctan 3(1-$ $\left.\lambda^{2}\right)$. In other words, each of the fixed points (38) and (39) appear twice as $\theta$ makes one revolution $(0 \rightarrow 2 \pi)$.

The short wavelength boundary $\bar{Z}=1$ also plays an important role. On $\bar{Z}=1$, using $\theta$ as the perturbation variable, the differential equations (34) and (36) simplify to

$$
\frac{d \Sigma_{\varphi}}{d \bar{N}}=0, \quad \frac{d \theta}{d \bar{N}}=-\cos ^{2} \theta .
$$

There are therefore two lines of (non-hyperbolic) fixed points at $\bar{Z}=1$ with constant $\Sigma_{\varphi}$ given by $\cos \theta=0$, i.e., $\theta=\pi / 2+n \pi, n=0,1$. Except at these fixed points, $\theta$ is monotonically decreasing on $\bar{Z}=1$ at constant $\Sigma_{\varphi}$. It follows that the invariant set $\bar{Z}=1$ is spanned by a family of heteroclinic cycles (concentric circles) given by $\Sigma_{\varphi}=$ const., with $-1 \leq \Sigma_{\varphi} \leq 1$. At $\theta=\pi / 2+n \pi$ equation (36) takes the form

$$
\left.\frac{d \theta}{d \bar{N}}\right|_{\cos \theta=0}=-(1-\bar{Z}),
$$

which implies that orbits in the vicinity of $\bar{Z}=1$ shadow the heteroclinic cycles and do not end at the fixed points on these cycles. Moreover, as we will see, asymptotics are associated with $\Sigma_{\varphi}=\lambda, \pm 1$, and hence it is only the heteroclinic cycles at these values of $\Sigma_{\varphi}$ which are of asymptotic relevance. We will denote these heteroclinic cycles by $\mathcal{H}_{\lambda}, \mathcal{H}_{ \pm 1}$, respectively (this use of $\mathcal{H}$ with subscripts should not be confused with $\mathcal{H}=a H)$.

In Fig. 1 we show the key invariant sets and fixed points in the state space $\mathcal{S}=\mathcal{B} \times \mathcal{P}$.

\subsection{Orbit structure of the background state space $\mathcal{B}$}

The background state space $\mathcal{B}$ with coordinates $\left(\Sigma_{\varphi}, \bar{Z}\right)$ is defined by the inequalities

$$
-1 \leq \Sigma_{\varphi} \leq 1, \quad 0 \leq \bar{Z} \leq 1 .
$$

The differential equations that define a dynamical system on $\mathcal{B}$ are the first two equations of the system (34). In the background it is convenient to use the usual $e$-fold time $N$ instead of the modified time $\bar{N}$, leading to the following equations:

$$
\begin{aligned}
\Sigma_{\varphi}^{\prime} & =3\left(1-\Sigma_{\varphi}^{2}\right)\left(\lambda-\Sigma_{\varphi}\right), \\
\bar{Z}^{\prime} & =2\left(3 \Sigma_{\varphi}^{2}-1\right) \bar{Z}(1-\bar{Z}) .
\end{aligned}
$$




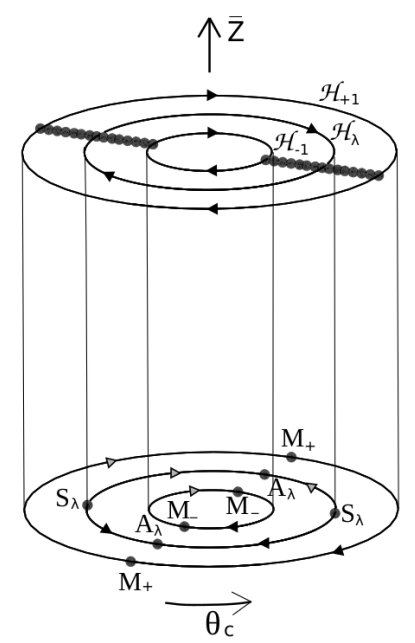

Figure 1: Invariant sets and fixed points in the compact state space $\mathcal{S}=\mathcal{B} \times \mathcal{P}$. The three coaxial cylinders whose common axis is the $\bar{Z}$ - axis with $0 \leq \bar{Z} \leq 1$ are the invariant cylinders $\Sigma_{\varphi}=-1, \lambda, 1$. The isolated fixed points $\mathrm{A}_{\lambda}, \mathrm{S}_{\lambda}$ and $\mathrm{M}_{ \pm 1}$ lie in the long wavelength boundary $\bar{Z}=0$ and the short wavelength boundary $\bar{Z}=1$ contains the heteroclinic cycles $\mathcal{H}_{-1}, \mathcal{H}_{\lambda}, \mathcal{H}_{1}$.

The fixed points of this dynamical system can be found by inspection. If $\lambda \neq 1 / \sqrt{3}$ there are six isolated fixed points, which lie on the boundary of the rectangle 42 ):

$$
\begin{array}{ll}
\bar{Z}=0, & \Sigma_{\varphi}= \pm 1, \lambda, \\
\bar{Z}=1, & \Sigma_{\varphi}= \pm 1, \lambda .
\end{array}
$$

The fixed points $\bar{Z}=0, \Sigma_{\varphi}= \pm 1$ are local sources and form the past attractor, while the fixed points $\bar{Z}=1, \Sigma_{\varphi}= \pm 1$ are saddles for all values of $\lambda$.

The future attractor depends on the value of the parameter $\lambda$, and is given by the following two fixed points:

$$
\begin{aligned}
\text { i) } \quad \bar{Z}=0, \quad \Sigma_{\varphi}=\lambda, \quad \text { a sink if } \quad 0 \leq \lambda<\frac{1}{\sqrt{3}}, \\
\text { ii) } \quad \bar{Z}=1, \quad \Sigma_{\varphi}=\lambda, \quad \text { a sink if } \quad \frac{1}{\sqrt{3}}<\lambda<1 .
\end{aligned}
$$

The line $\Sigma_{\varphi}=\lambda \neq 1 / \sqrt{3}$ is a special orbit joining the fixed points i) and ii). If $\lambda=1 / \sqrt{3}$ (and therefore $q=0$ ) this line becomes a line of fixed points, which corresponds to a bifurcation that transfers stability from i) to ii) as $\lambda$ increases.

The qualitative behaviour of typical and special orbits in the two cases $0 \leq \lambda<$ $1 / \sqrt{3}$ and $1 / \sqrt{3}<\lambda<1$ is illustrated in Fig. 2 .

Referring to Fig. 2 we can summarize the asymptotic behaviour of the orbits in the background state space $\mathcal{B}$ for $\lambda \neq 1 / \sqrt{3}$ as follows. For evolution into the future 


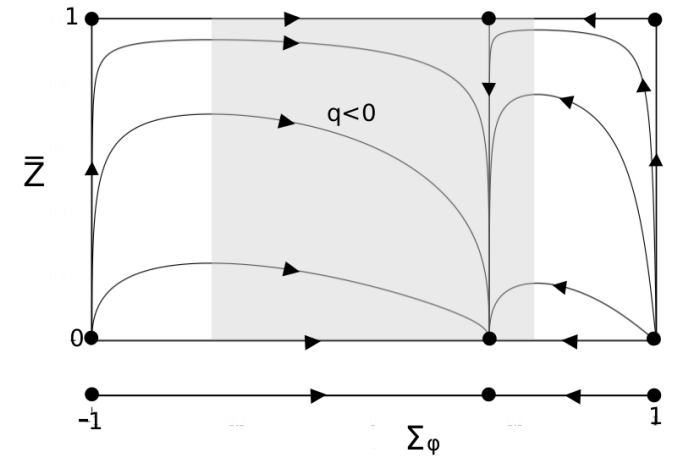

(a) $\lambda=1 / \sqrt{6}$

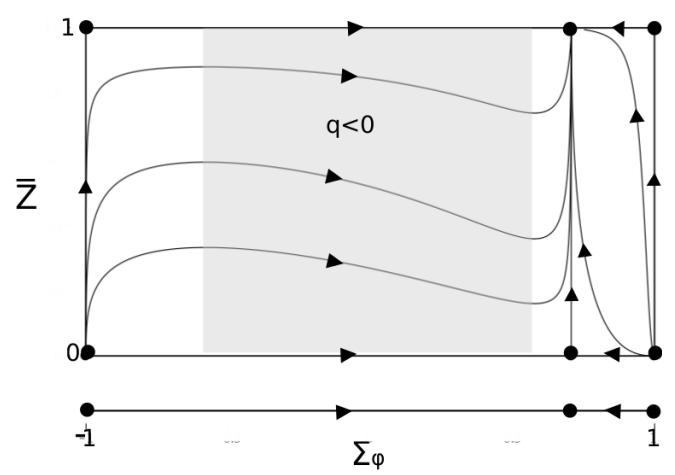

(b) $\lambda=1 / \sqrt{2}$

Figure 2: Orbits and fixed points in the background state space $\mathcal{B}$ using $e$-fold time $N$, with $0 \leq \lambda<1 / \sqrt{3}$, represented by $\lambda=1 / \sqrt{6}$, in panel (a), and $1 / \sqrt{3}<\lambda<1$, represented by $\lambda=1 / \sqrt{2}$, in panel (b). The shaded regions denote when $q<0$ and where $Z$ and $\bar{Z}$ are monotonically decreasing.

for all orbits with $\Sigma_{\varphi}^{2}<1$ we have

$$
\begin{aligned}
& \lim _{N \rightarrow \infty} \Sigma_{\varphi}=\lambda, \\
& \lim _{N \rightarrow \infty} \bar{Z}=0, \quad \text { if } \quad 0 \leq \lambda<1 / \sqrt{3}, \\
& \lim _{N \rightarrow \infty} \bar{Z}=1, \quad \text { if } \quad 1 / \sqrt{3}<\lambda<1 .
\end{aligned}
$$

Into the past all orbits with $\Sigma_{\varphi} \neq \lambda$ satisfy,

$$
\lim _{N \rightarrow-\infty} \Sigma_{\varphi}^{2}=1, \quad \lim _{N \rightarrow-\infty} \bar{Z}=0,
$$

while the special case $\Sigma_{\varphi}=\lambda$ results in

$$
\begin{gathered}
\lim _{N \rightarrow-\infty} \bar{Z}=1, \quad \text { if } \quad 0 \leq \lambda<1 / \sqrt{3}, \\
\lim _{N \rightarrow-\infty} \bar{Z}=0, \quad \text { if } \quad 1 / \sqrt{3}<\lambda<1 .
\end{gathered}
$$

For the special case $\Sigma_{\varphi}= \pm 1$ we have

$$
\lim _{N \rightarrow \infty} \bar{Z}=1, \quad \lim _{N \rightarrow-\infty} \bar{Z}=0, \quad \text { for all } \lambda .
$$

In the next section we will use these results to determine the asymptotic behaviour of the orbits in the full state space $\mathcal{B} \times \mathcal{P}$.

Before continuing we make some remarks relating our description of the background dynamics to previous research. The first paper to give a dynamical systems analysis of a scalar field with exponential potential is Halliwell (1987) [17], who used the background scalar field $\varphi$ and an exponential representation of the cosmological scale factor as variables.14 From our perspective, the limitation of his approach is

\footnotetext{
${ }^{14}$ In his figure 2, the flat background case is represented by the bold face hyperbola, and this corresponds to our background state space on the $\Sigma_{\varphi}$-axis shown in our Figure 2 since the exponential scale factor variable decouples in the flat case. The fixed point $\mathrm{A}$ in his figure 2 is the future attractor, which corresponds to our fixed point with $\Sigma_{\varphi}=\lambda$.
} 
that the state space is unbounded. The bounded variable $\Sigma_{\varphi}$ that we are using is not new. It has been introduced before in the context of a scalar field with an exponential potential by Coley et al (1997) [18] and by Copeland et al (1998) [19]. Both these references considered more general background problems than the present one: Coley et al studied anisotropic Bianchi models and Copeland et al added a perfect fluid with a linear equation of state to the scalar field. The variable $d \varphi / d N$, which is proportional to $\Sigma_{\varphi}$, is now commonly used to describe scalar fields in cosmology. ${ }^{15}$

Our state space $\mathcal{B}$ differs in an important way from [17, 19], in that it also describes the dynamics of the variable $Z=k^{2} / \mathcal{H}^{2}$, compactified to give $\bar{Z}$, whose evolution is determined by $\Sigma_{\varphi}$ through the differential equation (43b), which leads to the two dimensional state space in our figure 2 . This yields a complete description of the dynamics of a scalar field with exponential potential in a flat FL background needed to determine the evolution of perturbations on this background, which is the main goal of this paper. This representation of the dynamics, which to the best of our knowledge is new, has several advantages. It shows directly in which regions of the state space there is accelerated expansion (the shaded regions in figure 2, in which $q=3 \Sigma_{\varphi}^{2}-1<0$ and where thereby $Z$ and $\bar{Z}$ are monotonically decreasing), and highlights the physical interpretation of the parameter $\lambda$ in the potential. Referring to our figure 2 , if $\lambda<1 / \sqrt{3}$ then all orbits eventually enter and remain in the region of accelerated expansion and thus the orbits describe universes that undergo future accelerated expansion. On the other hand if $\lambda<1 / \sqrt{3}$ orbits that are past asymptotic to $\Sigma_{\varphi}=1$ do not enter the shaded region and hence never undergo accelerated expansion, while orbits that are past asymptotic to $\Sigma_{\varphi}=-1$ enter and then leave the shaded region and hence undergo transient accelerated expansion. We note that the general orbits in Figure 2 have been given explicitly in parametric form $H(u), \varphi(u)$, where $u$ is a parameter, by Salopek and Bond (1990) [23], and analyzed qualitatively (see equations (3.2) and their figure 1). Our state space picture shows this behaviour directly.

\subsection{General and special orbits in $\mathcal{B} \times \mathcal{P}$}

Since the full state space has a product structure $\mathcal{S}=\mathcal{B} \times \mathcal{P}$, the limits of $\Sigma_{\varphi}$ and $\bar{Z}$ as $N \rightarrow \pm \infty$ given by equations (46), (47), (48) and (49), which we derived in the background state space $\mathcal{B}$ (see also Fig. 2), are also valid in $\mathcal{B} \times \mathcal{P}$, and we can use these results to determine the asymptotic behaviour of the orbits in the full state space. We still limit the discussion to $0 \leq \lambda<1$, and we also exclude the bifurcation value $\lambda=1 / \sqrt{3}$. The restrictions imposed in deriving (46) and (47) require that we consider separately the generic orbits and the special orbits that satisfy $\Sigma_{\varphi}=\lambda$ and $\Sigma_{\varphi}= \pm 1$ with constant deceleration parameter $q$. We refer to the case $\Sigma_{\varphi}=\lambda$ as the (interior) scale-invariant orbits ${ }^{16}$ and the orbits with $\Sigma_{\varphi}= \pm 1$ as the massless

\footnotetext{
${ }^{15}$ See for example, Urena-Lopez (2012) [20, equation (2.3), Tsujikawa (2013) 21, equation (16) and Alho and Uggla (2015) [22, equation (8).

${ }^{16}$ The solutions corresponding to orbits with $\Sigma_{\varphi}=\lambda>0$ are invariant under constant conformal scalings and admit a homothetic Killing vector field. The orbits with $\Sigma_{\varphi}=\lambda=0$ correspond to the one-parameter family of de Sitter solutions, where a constant scaling scales the dimensional cosmological constant that is parametrizing the family of solutions. Constant conformal scalings thereby result in the same solution when $\lambda>0$ while they lead to a new member in the same
} 
scalar field orbits (although they also correspond to scale-invariant solutions). On recalling that $q=3 \Sigma_{\varphi}^{2}-1$, we have

$$
q=3 \lambda^{2}-1 \text { in the scale-invariant case; } \quad q=2 \text { in the massless scalar field case. }
$$

First as regards the generic orbits it follows from equations (46) and (47) that

Gi) all generic orbits are past asymptotic to one of the fixed points $\mathrm{M}_{ \pm}$, which form the past attractor of the dynamical system,

Gii) if $\lambda<1 / \sqrt{3}$ then a two parameter family of orbits is future asymptotic to the fixed point $A_{\lambda}$, which forms the future attractor; in addition a one-parameter family of orbits is future asymptotic to the fixed point $S_{\lambda}$,

Giii) if $\lambda>1 / \sqrt{3}$ then all orbits are future asymptotic to the heteroclinic cycle $\mathcal{H}_{\lambda}$, which forms the future attractor.

We give some details of the derivation of the result Gii) at the end of this section.

We now describe the acceleration/deceleration properties of these generic models. ${ }^{17}$ Since $q=3 \Sigma_{\varphi}^{2}-1$ we can draw the following conclusions by referring to Fig. 2 and using the results Gi)-Giii) above:

i) If $\lambda<1 / \sqrt{3}$, then all models undergo future acceleration,

ii) If $\lambda>1 / \sqrt{3}$ then all models undergo future deceleration. However, there are two subcases. Orbits that are past asymptotic to $\mathrm{M}_{-}$yield models that undergo transient acceleration, i.e. they have a finite epoch during which $q<0$, see Fig. 2 ; orbits that are past asymptotic to $\mathrm{M}_{+}$yield models that are forever decelerating, i.e. $q>0$ at all times.

Fig. 3 shows examples of generic orbits in the two cases $0 \leq \lambda<1 / \sqrt{3}$ and $1 / \sqrt{3}<\lambda<1$.

Before continuing we make a remark concerning the physical interpretation of Figure 3. It is helpful to think how the orbits are generated numerically. One chooses initial values for $\Sigma_{\varphi}, \bar{Z}$ and $\theta$ and then integrates numerically forwards and backwards to generate an orbit 18 One can use the initial value of $\bar{Z}$ in conjunction

family of solutions when $\lambda=0$. The close connection between the two classes is illustrated by that both $\lambda>0$ and $\lambda=0$ yield constant values for dimensionless scalars such as $\Sigma_{\varphi}$ and $q$. For simplicity we will therefore also refer to $\lambda=0$ as belonging to the scale-invariant case.

${ }^{17}$ The region of state space in which there is accelerated expansion is a cylindrical shell in the state space $\mathcal{S}=\mathcal{B} \times \mathcal{P}$, given by the inequalities $-1 / \sqrt{3}<\Sigma_{\varphi}<1 / \sqrt{3}$. This region can be visualized by rotating the shaded region in figure 2 about a line $\Sigma_{\varphi}=b<-1$, as described at the beginning of section 4.1 .

${ }^{18}$ The usual way to numerically integrate the perturbation equations for scalar fields is to first integrate the background equations, and then use the output to integrate the perturbed KleinGordon equation to determine the real and imaginary parts of the Fourier transform of the scalar field perturbation, for a given wave number $k$. One is thereby treating the perturbed Klein-Gordon equation as a non-autonomous differential equation, in contrast to our dynamical systems approach. See for example Huston and Malik (2009) 24] (this paper also covers second order perturbations) and Martin and Ringeval (2006) [25] (see section 4.1). 


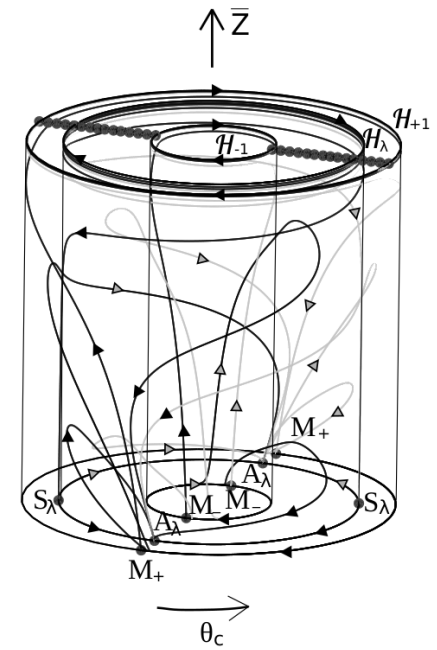

(a) $\lambda=1 / \sqrt{6}$

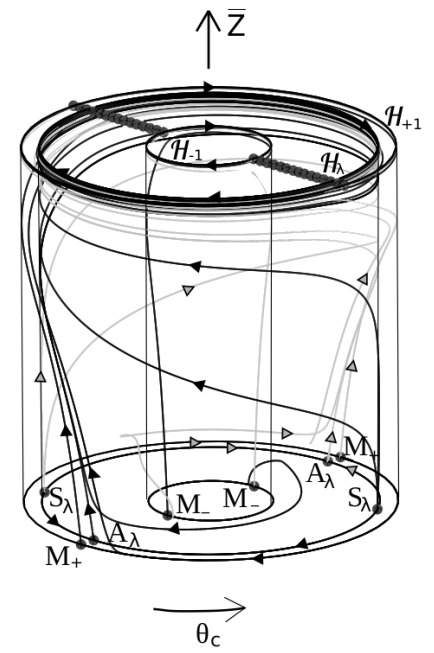

(b) $\lambda=1 / \sqrt{2}$

Figure 3: Representative orbits on the state space $\mathcal{S}=\mathcal{B} \times \mathcal{P}$ as described in results Gi)-Giii), with $0 \leq \lambda<1 / \sqrt{3}$, illustrated by $\lambda=1 / \sqrt{6}$, in panel (a), and $1 / \sqrt{3}<\lambda<1$, illustrated by $\lambda=1 / \sqrt{2}$, in panel (b).

with equation (31) to determine the wave number $k$ in terms of the value of the Hubble scalar $H_{0}$ at the initial time $a_{0}=1, N_{0}=0$ :

$$
\bar{Z}_{0}=\frac{k^{2}}{k^{2}+\mathcal{H}_{0}^{2}}=\frac{k^{2}}{k^{2}+H_{0}^{2}} .
$$

In this way one can generate a family of orbits corresponding to different wave numbers.

Next, as regards the interior scale-invariant orbits $\left(\Sigma_{\varphi}=\lambda<1\right)$ it follows from equation (48) that

Ai) if $0 \leq \lambda<1 / \sqrt{3}$ (i.e. $q<0$ ) then a scale-invariant orbit is past asymptotic to the heteroclinic cycle $\mathcal{H}_{\lambda}$ and is future asymptotic to the fixed point $A_{\lambda}$, apart from one exceptional orbit that is future asymptotic to the fixed point $\mathrm{S}_{\lambda}$,

Aii) if $1 / \sqrt{3}<\lambda<1$ (i.e. $q>0$ ) then a scale-invariant orbit is future asymptotic to the heteroclinic cycle $\mathcal{H}_{\lambda}$ and is past asymptotic to the fixed point $\mathrm{S}_{\lambda}$, apart from one exceptional orbit that is past asymptotic to the fixed point $A_{\lambda}$.

Representative scale-invariant orbits are depicted in Fig. 4 (recall that there are two copies of fixed points and orbits when using $\theta$ instead of $y$, related to each other by $\theta \rightarrow \theta+\pi)$.

Finally, as regards the massless scalar field orbits $\left(\Sigma_{\varphi}= \pm 1\right)$ it follows from equations 49 that such an orbit is past asymptotic to one of the fixed points $\mathrm{M}_{ \pm}$, and is future asymptotic to one of the heteroclinic cycles $\mathcal{H}_{ \pm 1}$. Representative massless scalar field orbits are depicted in Fig. 5.

In deriving the result Gii), the limits (46) in $\mathcal{B}$ imply that $\lim _{N \rightarrow \infty} \Sigma_{\varphi}=\lambda$, and $\lim _{N \rightarrow \infty} \bar{Z}=0$ in the state space $\mathcal{S}=\mathcal{B} \times \mathcal{P}$. It follows that the orbits in question 


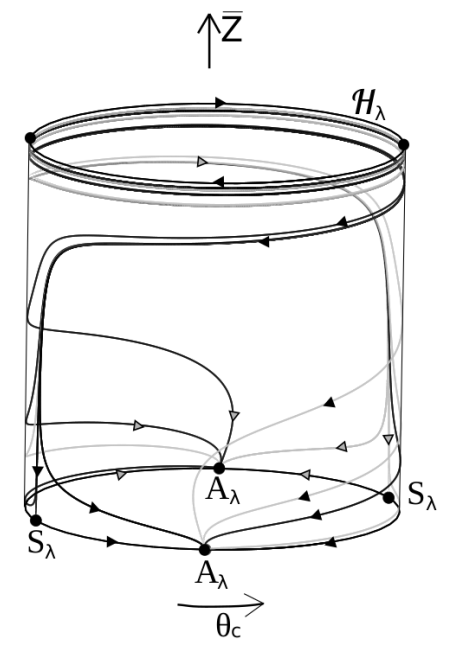

(a) $\lambda=1 / \sqrt{6}$

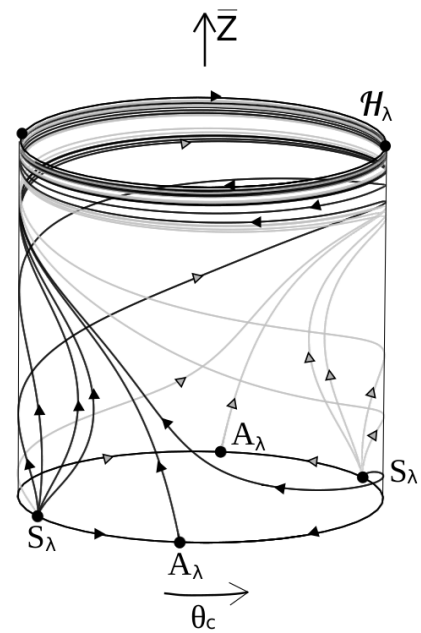

(b) $\lambda=1 / \sqrt{2}$

Figure 4: Representative orbits on the invariant set $\Sigma_{\varphi}=\lambda$ : Ai) for which $0 \leq \lambda<$ $1 / \sqrt{3}$ is illustrated by $\lambda=1 / \sqrt{6}$ in panel (a), while Aii) for which $1 / \sqrt{3}<\lambda<1$ is illustrated by $\lambda=1 / \sqrt{2}$ in panel (b).

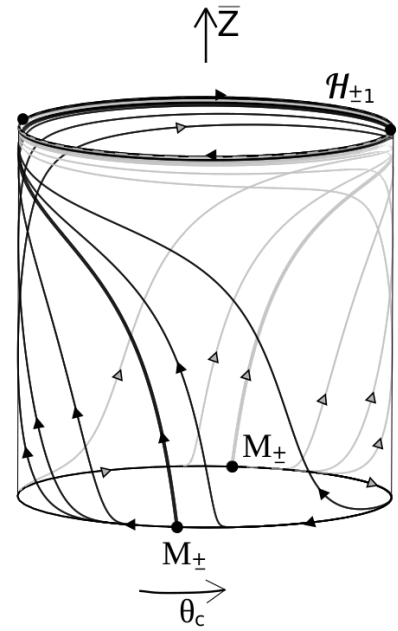

Figure 5: Representative orbits on the massless scalar field boundary for any value of $\lambda$ (the orbits on the two components $\Sigma_{\varphi}=-1$ and $\Sigma_{\varphi}=+1$ are identical).

are future asymptotic to the one dimensional invariant set $\Sigma_{\varphi}=\lambda, \bar{Z}=0$ (a circle). Since this circle contains the two hyperbolic fixed points $A_{\lambda}$ and $S_{\lambda}$, the orbits in question must be future asymptotic to one of these fixed points. The final step is to note that in this case $A_{\lambda}$ is a sink and $S_{\lambda}$ is a saddle with a two dimensional stable manifold.

The local stability properties of $\mathrm{A}_{\lambda}$ and $\mathrm{S}_{\lambda}$ also yield the local stability properties for $\mathrm{Ai}$ ) and $\mathrm{Aii}$ ): in $\mathrm{Ai}$ ) $\mathrm{A}_{\lambda}$ is a sink and $\mathrm{S}_{\lambda}$ is a saddle on the interior scale-invariant set $\Sigma_{\varphi}=\lambda$, while in Aii) $\mathrm{S}_{\lambda}$ is a source and $\mathrm{A}_{\lambda}$ is a saddle, see Fig. 4 . 


\subsection{Asymptotic expansions}

In this subsection we will give asymptotic expansions for $\Sigma_{\varphi}, Z, y$ and $\varphi_{\mathrm{c}}$ in terms of the $e$-fold time $N$ in the vicinity of the fixed points in the long wavelength boundary set $Z=0$. When it comes to $\varphi_{\mathrm{c}}$, we will focus on the future accelerating case $0 \leq \lambda<1 / \sqrt{3}$, for which we need $\varphi_{\mathrm{c}}$ in section 6 .

\section{Asymptotics for the fixed point $A_{\lambda}$}

We have shown that for generic orbits with $0 \leq \lambda<1 / \sqrt{3}$ the future attractor is the fixed point $\mathrm{A}_{\lambda}: \Sigma_{\varphi}=\lambda, Z=0, y=0$, with $q=3 \lambda^{2}-1<0$. Solving the evolution equations 29 to leading order in the vicinity of this fixed point yields the following expressions:

$$
\begin{aligned}
\Sigma_{\varphi} & \approx \lambda+C_{1} e^{-3\left(1-\lambda^{2}\right) N}, \\
Z & \approx C_{2} e^{-2\left(1-3 \lambda^{2}\right) N}, \\
y & \approx C_{3} e^{-3\left(1-\lambda^{2}\right) N}-\frac{C_{2}}{1+3 \lambda^{2}} e^{-2\left(1-3 \lambda^{2}\right) N},
\end{aligned}
$$

as $N \rightarrow+\infty$, where $C_{1}, C_{2} \geq 0$, and $C_{3}$ are constants, where $C_{2}>0$ when $k^{2}>0$. The scale-invariant orbits are given by $C_{1}=0$. It follows from (52c) and (26) that for the orbits, which are all converging to $\mathrm{A}_{\lambda}$,

$$
\varphi_{\mathrm{c}} \approx C_{\varphi}^{+}\left(1-\frac{C_{3}}{3\left(1-\lambda^{2}\right)} e^{-3\left(1-\lambda^{2}\right) N}+\frac{C_{2}}{2\left(1-9 \lambda^{4}\right)} e^{-2\left(1-3 \lambda^{2}\right) N}\right),
$$

where $\lim _{N \rightarrow+\infty} \varphi_{\mathrm{c}}=C_{\varphi}^{+}$when $0 \leq \lambda<1 / \sqrt{3}$. For future decelerating models $(1 / \sqrt{3}<\lambda<1)$ there is a unique orbit in the invariant set $\Sigma_{\varphi}=\lambda$, characterized by $C_{1}=C_{3}=0$ that is past asymptotic to $A_{\lambda}$.

\section{Asymptotics for the fixed point $S_{\lambda}$}

The linear solution describing the solutions in the vicinity of $S_{\lambda}$ is given by

$$
\begin{aligned}
\Sigma_{\varphi} & =\lambda+C_{1} e^{-3\left(1-\lambda^{2}\right) N} \\
Z & =C_{2} e^{-2\left(1-3 \lambda^{2}\right) N} \\
y & =-3\left(1-\lambda^{2}\right)+3 \lambda C_{1} e^{-3\left(1-\lambda^{2}\right) N}+\frac{C_{2}}{5-9 \lambda^{2}} e^{-2\left(1-3 \lambda^{2}\right) N}+C_{3} e^{3\left(1-\lambda^{2}\right) N},
\end{aligned}
$$

where again $C_{2}>0$ when $k^{2}>0$. This implies that for the future accelerating models $0 \leq \lambda<1 / \sqrt{3}$ there is a one-parameter family of orbits that are future asymptotic to the hyperbolic saddle point $\mathrm{S}_{\lambda}$, characterized by $C_{3}=0$, where the orbit with $C_{1}=0$ lies in the interior scale-invariant set $\Sigma_{\varphi}=\lambda$. In this case

$$
\varphi_{\mathrm{c}} \approx C_{\varphi}^{+} e^{-3\left(1-\lambda^{2}\right) N}\left(1-\frac{\lambda C_{1}}{1-\lambda^{2}} e^{-3\left(1-\lambda^{2}\right) N}-\frac{C_{2}}{2\left(1-3 \lambda^{2}\right)\left(5-9 \lambda^{2}\right)} e^{-2\left(1-3 \lambda^{2}\right) N}\right),
$$

where $\lim _{N \rightarrow+\infty} \varphi_{\mathrm{c}}=0$. For the future decelerating models with $1 / \sqrt{3}<\lambda<1$ there is a one-parameter family of orbits that are past asymptotic to $\mathrm{S}_{\lambda}$ in the interior scale-invariant set $\Sigma_{\varphi}=\lambda$, characterized by $C_{1}=0$. 


\section{Asymptotics for the fixed points $\mathrm{M}_{ \pm}$}

We have shown that for generic orbits the past attractor is the pair of fixed points $\mathrm{M}_{ \pm}: \Sigma_{\varphi}= \pm 1, \bar{Z}=Z=0, y=0$. Solving the evolution equations (29) to leading order in the vicinity of these fixed points results in

$$
\begin{aligned}
\Sigma_{\varphi} & \approx \pm\left(1-C_{1} e^{6(1 \mp \lambda) N}\right), \\
Z & \approx C_{2} e^{4 N}, \\
y & \approx \frac{C_{3}}{1+C_{3} N}-\frac{C_{2}}{4} e^{4 N}-6(1 \mp \lambda) C_{1} e^{6(1 \mp \lambda) N},
\end{aligned}
$$

as $N \rightarrow-\infty$, where $C_{1} \geq 0, C_{2} \geq 0, C_{3}$ are constants, with $C_{2} \propto k^{2}$ where $C_{2}>0$ when $k^{2}>0$, and where we have used the translation freedom in $N$ to set a constant $A$ in a term $C_{3} /\left(A+C_{3} N\right)$ to one. Note that $C_{3}=0$ describes the two-dimensional unstable manifolds of the fixed points $\mathrm{M}_{ \pm}$, while the power law approach to zero in the $C_{3}$ term is due to presence of a zero eigenvalue and resulting centre manifold. It follows from (56c) and (26) that to leading order

$$
\varphi_{\mathrm{c}} \approx C_{\varphi}^{-}\left(1+C_{3} N\right)\left(1-\frac{C_{2}}{16} e^{4 N}-C_{1} e^{6(1 \mp \lambda) N}\right),
$$

where $\lim _{N \rightarrow-\infty} \varphi_{\mathrm{c}}=C_{\varphi}^{-}$when $C_{3}=0$, while $\varphi_{\mathrm{c}}$ becomes unbounded toward the past when $C_{3} \neq 0$.

\section{Explicit solutions with constant deceleration pa- rameter}

The Klein-Gordon equation (20) for a perturbed scalar field with exponential potential has been solved explicitly for $\varphi_{\mathrm{c}}$ subject to the assumption that the scale factor $a$ has a power law dependence on conformal time, and thereby a constant deceleration parameter $q$ (see footnote 1 for references). In this case it is convenient to introduce a new variable

$$
v=a \varphi_{\mathrm{c}},
$$

and to use conformal time $\eta$ instead of $e$-fold time $N{ }^{19}$

Recall that $\varphi_{\mathrm{c}}$ is the complex Fourier coefficient of the scalar field perturbation in the uniform curvature gauge. Note that we have dropped the index $k$ on $\varphi_{\mathrm{c}, k}$, and where we now similarly drop the index $k$ on $v_{k}$. Making the above changes transform equation (20) into the following Bessel equation for the function $v{ }^{20}$

$$
\partial_{\eta}^{2} v+\left(k^{2}-\left(\nu^{2}-\frac{1}{4}\right) \eta^{-2}\right) v=0,
$$

\footnotetext{
${ }^{19}$ In making the transition from $N$ to $\eta$ we use the relations $\partial_{\eta}=\mathcal{H} \partial_{N}, \partial_{\eta}^{2}=\mathcal{H}^{2}\left(\partial_{N}^{2}-q \partial_{N}\right)$, and equation 61.

${ }^{20}$ Since $q=3 \Sigma_{\varphi}^{2}-1$ by $(10), q^{\prime}=0 \Longrightarrow \Sigma_{\varphi}^{\prime}=0$, which gives $\left(1-\Sigma_{\varphi}^{2}\right)\left(\lambda-\Sigma_{\varphi}\right)=0$ using 12b). These results reduce equation (20) to $\partial_{N}^{2} \varphi_{\mathrm{c}}+(2-q) \partial_{N} \varphi_{\mathrm{c}}+k^{2} \mathcal{H}^{-2} \varphi_{\mathrm{c}}=0$. The next stage in the derivation is $\partial_{\eta}^{2} \varphi_{\mathrm{c}}+2 \mathcal{H} \partial_{\eta} \varphi_{\mathrm{c}}+k^{2} \varphi_{\mathrm{c}}=0$.
} 
where the index $\nu$ is determined by $q$ according to

$$
\nu=\frac{2-q}{2|q|} .
$$

In analyzing the solutions we need the following properties of conformal time that follow from the assumption that $q$ is constant:

$$
\mathcal{H} \eta=q^{-1}, \quad \eta / \eta_{0}=e^{q N}, \quad \mathcal{H}=\mathcal{H}_{0} e^{-q N} .
$$

Since we are considering expanding models $(\mathcal{H}>0), \eta$ has the same sign as $q$. If $q<0$, then $-\infty<\eta<0$ and the limit $\eta \rightarrow 0^{-}$describes late times since $N \rightarrow \infty$ as $\eta \rightarrow 0^{-}$, while the limit $\eta \rightarrow-\infty$ describes early times since $N \rightarrow-\infty$ as $\eta \rightarrow-\infty$. If $q>0$, then $0<\eta<\infty$ where the limit $\eta \rightarrow 0^{+}$describes early times since $N \rightarrow-\infty$ as $\eta \rightarrow 0^{+}$, while the limit $\eta \rightarrow \infty$ describes late times since $N \rightarrow \infty$ as $\eta \rightarrow \infty$.

The general solution of Bessel's equation (59) is given in terms of Bessel functions by

$$
v=\sqrt{k|\eta|}\left(C_{+} J_{\nu}(k|\eta|)+C_{-} Y_{\nu}(k|\eta|)\right),
$$

where $C_{+}$and $C_{-}$are complex constants that depend on $k$, and $v=a \varphi_{\mathrm{c}}$.

The reduced dynamical variable $y$ is defined in equation (24) by $y=f^{\prime} / f$, where $f$ is a real function, either the real or imaginary part of $\varphi_{\mathrm{c}}$. The function $y=f^{\prime} / f$ can be expressed in terms of the corresponding function $v=a f$ according to:

$$
y=\frac{f^{\prime}}{f}=\frac{v^{\prime}}{v}-1=\frac{\partial_{\eta} v}{\mathcal{H} v}-1=\frac{q \eta \partial_{\eta} v}{v}-1
$$

where we have used the first equation in (61). This expression for $y$, with $v$ being any real function contained in the general solution $(62)$, describes the one-parameter ${ }^{21}$ family of orbits of the dynamical system for which $q$ is constant, in other words the scale-invariant and massless scalar field orbits. In order to complete the identification we need to determine the asymptotic form of $y$, given by $(62)$ and $(63)$ as $\eta \rightarrow 0$ and $\eta \rightarrow \pm \infty$.

The asymptotic form of the Bessel functions is

$$
\begin{gathered}
J_{\nu}(z) \sim z^{\nu}, \quad \text { for } \quad \nu \geq 0 \text { as } z \rightarrow 0, \\
Y_{\nu}(z) \sim z^{-\nu}, \text { for } \quad \nu>0, \quad Y_{0}(z) \sim \ln z, \quad \text { as } z \rightarrow 0,
\end{gathered}
$$

and

$$
J_{\nu}(z)+i Y_{\nu}(z) \approx \sqrt{\frac{2}{\pi z}} \exp \left[i\left(z-\frac{1}{4}(2 \nu+1) \pi\right)\right], \quad \text { as } \quad z \rightarrow \infty,
$$

where $\sim$ means proportional to, i.e., we are dropping constant factors that depend on $\nu$.

It follows from (62) and (64) that for the scale-invariant orbits $\left(\Sigma_{\varphi}=\lambda<1\right)$ there are two cases as $|\eta| \rightarrow 0$. First the general case $C_{-} \neq 0$ in which $Y_{\nu}$ will be

\footnotetext{
${ }^{21}$ This expression for $y$ can be written in terms of only one parameter, either $C_{+} / C_{-}$or $C_{-} / C_{+}$, without loss of generality, when $C_{-} \neq 0$ or $C_{+} \neq 0$, respectively.
} 
the dominant term, and a special case $C_{-}=0$ in which $Y_{\nu}$ drops out making $J_{\nu}$ the dominant term. We now use (62), (63), and (64) to calculate the asymptotic form of $y$ :

$$
\begin{aligned}
& C_{-} \neq 0 \Longrightarrow v \sim|\eta|^{1 / 2-\nu} \Longrightarrow y \rightarrow q\left(\frac{1}{2}-\nu\right)-1, \quad \text { as } \quad|\eta| \rightarrow 0 \\
& C_{-}=0 \Longrightarrow v \sim|\eta|^{1 / 2+\nu} \Longrightarrow y \rightarrow q\left(\frac{1}{2}+\nu\right)-1, \quad \text { as } \quad|\eta| \rightarrow 0 .
\end{aligned}
$$

It follows from (59) that $q \nu=-\frac{1}{2}(2-q)$, if $q<0$, and $q \nu=\frac{1}{2}(2-q)$, if $q>0$. The final results when $|\eta| \rightarrow 0$ are:

$$
\begin{array}{ll}
\text { If } q<0 \text {, then } C_{-} \neq 0 \Longrightarrow y \approx 0, & C_{-}=0 \Longrightarrow y \approx-(2-q), \\
\text { If } q>0, \text { then } C_{-} \neq 0 \Longrightarrow y \approx-(2-q), & C_{-}=0 \Longrightarrow y \approx 0 .
\end{array}
$$

These limiting values are consistent with the fixed points in the interior scaleinvariant set $\Sigma_{\varphi}=\lambda$ that are given by $\mathrm{A}_{\lambda}: y=0$, and $\mathrm{S}_{\lambda}: y=-3\left(1-\lambda^{2}\right)=-(2-q)$. If $q<0$ this gives the evolution into the future and for $q>0$ the evolution into the past.

In the limit $|\eta| \rightarrow \infty$, it follows from equations (62) and (65) that $v \sim \exp ( \pm i k|\eta|)$ is sinusoidal. Thus if $q<0(q>0)$ the evolution into the past (future) is sinusoidal in terms of conformal time ${ }^{22}$ In our dynamical systems framework, however, this asymptotic behaviour is described by the shadowing of the heteroclinic cycle $\mathcal{H}_{\lambda}$, which is infinitely repetitive but not sinusoidal.

We have thus confirmed the asymptotic properties of the scale-invariant orbits:

Ai) Accelerating models $(q<0)$.

Orbits with $C_{-} \neq 0$ evolve from $\mathcal{H}_{\lambda}$ to $\mathrm{A}_{\lambda}$.

The exceptional orbit with $C_{-}=0$ evolves from $\mathcal{H}_{\lambda}$ to $\mathrm{S}_{\lambda}$.

Aii) Decelerating models $(q>0)$.

Orbits with $C_{-} \neq 0$ evolve from $\mathrm{S}_{\lambda}$ to $\mathcal{H}_{\lambda}$.

The exceptional orbit with $C_{-}=0$ evolves from the fixed point $A_{\lambda}$ to $\mathcal{H}_{\lambda}$.

For the massless scalar field orbits $(q=2)$ we have $\eta>0$ and the expression for $y$ is given by (62) and (63) with $\nu=0$. It follows from (64b) that

$$
\begin{aligned}
& C_{-} \neq 0 \Longrightarrow v \sim \sqrt{\eta} \ln \eta \Longrightarrow y \rightarrow 0, \quad \text { as } \quad \eta \rightarrow 0, \\
& C_{-}=0 \Longrightarrow v \sim \sqrt{\eta} \quad \Longrightarrow y \rightarrow 0, \quad \text { as } \quad \eta \rightarrow 0,
\end{aligned}
$$

the difference being that $y$ tends to zero logarithmically when $C_{-} \neq 0,(y \approx$ $2 / \ln \eta){ }^{23}$ Thus the massless scalar field orbits are past asymptotic to one of the fixed points $\mathrm{M}_{ \pm}$. As $\eta \rightarrow \infty$, equation (65) implies that $y$ is oscillatory. Since the massless scalar field orbits satisfy $\Sigma_{\varphi}= \pm 1$ this confirms that they are future asymptotic to one of the heteroclinic cycles $\mathcal{H}_{ \pm}$.

\footnotetext{
${ }^{22}$ It is of interest that in the case $q<0$ the initial condition for $v$ that leads to power law inflation and the derivation of the power spectrum for the perturbations is $v=(1 \sqrt{2 k}) \exp (-i k \eta)$, as $\eta \rightarrow-\infty$. See, for example Durrer (2008) [3], pages 112-114, in particular equation (3.47).

${ }^{23}$ The logarithmic approach to zero in $\eta$ is due to presence of a zero eigenvalue and resulting centre manifold for the fixed points $\mathrm{M}_{ \pm}$.
} 


\section{The comoving and uniform density curvature perturbations}

It is generally believed that the comoving curvature perturbation, $\mathcal{R}$, and the uniform density curvature perturbation, $\zeta$, are conserved quantities for long wavelength adiabatic perturbations. ${ }^{24}$ a feature that plays an important role in inflationary cosmology. This heuristic statement means that in a time interval during which $k / \mathcal{H} \ll 1$ these gauge invariants are approximately constant and in addition are approximately equal in value. This result applies to a barotropic perfect fluid and to a minimally coupled scalar field with arbitrary potential. However, it has been pointed out by Romano et al (2016) [5] that it breaks down toward the future in the special case of a scalar field with a constant potential, which is said to describe so-called ultra-slow roll inflation.

In this section we apply our dynamical system formulation to give a rigorous analysis of $\mathcal{R}$ and $\zeta$ in the present case of a minimally coupled scalar field with exponential potential, and restricting our attention to future accelerating models $(0 \leq \lambda<1 / \sqrt{3}$, with $\lambda=0$ giving a constant potential). Earlier we showed that for these models as $N \rightarrow \pm \infty$ almost all orbits are asymptotic to fixed points in the long wavelength boundary $Z=0$. The only exceptions are the orbits that describe models with a constant deceleration parameter. These orbits, which lie in the invariant sets $\Sigma_{\varphi}=\lambda$ and $\Sigma_{\varphi}= \pm 1$, differ in that they are past/future asymptotic to the short wavelength boundary $\bar{Z}=1$, respectively.

We proceed by using previous results to derive asymptotic expressions for $\mathcal{R}$ and $\zeta$ on approach to the fixed points labelled $\mathrm{M}_{ \pm}, \mathrm{A}_{\lambda}, \mathrm{S}_{\lambda}$, i.e., as $N \rightarrow \pm \infty$ and $Z \rightarrow 0$. In view of the heuristic conservation property of $\mathcal{R}$ and $\zeta$ one might conjecture that the $\operatorname{limits}_{\lim _{N \rightarrow \pm \infty}} \mathcal{R}$ and $\lim _{N \rightarrow \pm \infty} \zeta$ would be finite and equal. However, we have found that this does not hold in general, since in two cases $\mathcal{R}$ and $\zeta$ become unbounded in the limit. In the first case, the conjecture fails for generic orbits into the past for $\lambda$ satisfying $0 \leq \lambda<1 / \sqrt{3}$, and in the second case for generic orbits into the future, but only for the ultra slow-roll case $\lambda=0$. In both cases, however, there are non-generic orbits for which the conjecture holds. In the first case we note that generic orbits are past asymptotic to one of the fixed points $\mathrm{M}_{+}$or $\mathrm{M}_{-}$and lie in the centre manifold of one of these fixed points. However, the conjecture does hold for orbits that lie in the unstable manifold of these fixed points, which is of one dimension less than the centre manifold. In the second case we note that generic orbits with $\lambda=0$ are asymptotic to the fixed point $\mathrm{A}_{0}$ into the future while the conjecture does hold for a one-parameter family of orbits that is asymptotic to $\mathrm{S}_{0}{ }^{25}$

To justify the above claims we derive asymptotic expressions for $\mathcal{R}$ and $\zeta$ as $N \rightarrow \pm \infty$. We begin with the following relations, working in the total matter (comoving) gauge:

$$
\mathcal{R} \equiv \psi_{\mathrm{v}}, \quad \zeta \equiv \psi_{\rho}=\psi_{\mathrm{v}}-\frac{1}{3} \boldsymbol{\delta}_{\mathrm{v}},
$$

\footnotetext{
${ }^{24}$ For the definition of these quantities in terms of gauge invariants, and a brief history with other references, see for example Uggla and Wainwright (2019) [26, section 4.

${ }^{25}$ The result of [5] concerning future non-conservation of $\mathcal{R}$ and $\zeta$ applies to the generic orbits with $\lambda=0$.
} 
where the second equation is the change of gauge formula for the curvature perturbation $\psi{ }^{26}$ For a perturbed scalar field one can show that ${ }^{27}$

$$
\psi_{\mathrm{v}}=\frac{\varphi_{\mathrm{c}}}{\varphi_{0}^{\prime}}, \quad \boldsymbol{\delta}_{\mathrm{v}}=\psi_{\mathrm{v}}^{\prime},
$$

We substitute (70) into (69) and use the dynamical system variables $y=\varphi_{\mathrm{c}}^{\prime} / \varphi_{\mathrm{c}}$ and $\varphi_{0}^{\prime}=\sqrt{6} \Sigma_{\varphi}$. After rearranging we obtain ${ }^{28}$

$$
\mathcal{R}=\frac{1}{\sqrt{6}}\left(\frac{\varphi_{\mathrm{c}}}{\Sigma_{\varphi}}\right), \quad \zeta=\mathcal{R}\left(1-\frac{1}{3}\left(y-\frac{\Sigma_{\varphi}^{\prime}}{\Sigma_{\varphi}}\right)\right)=\mathcal{R}-\frac{1}{3} \mathcal{R}^{\prime} .
$$

We can now derive asymptotic expressions for $\mathcal{R}$ and $\zeta$ in the neighbourhood of the fixed points for $\mathrm{M}_{ \pm}, \mathrm{A}_{\lambda}$ and $\mathrm{S}_{\lambda}$ using the asymptotic expressions for $y, \varphi_{\mathrm{c}}$ and $\Sigma_{\varphi}$ given in the previous section.

We first consider orbits approaching the fixed points $\mathrm{M}_{ \pm}$into the past. Keeping only the past dominant term ${ }^{29}$ in the expansions in section 4.4 we obtain the relations:

$$
\begin{aligned}
& \varphi_{\mathrm{c}} \approx C_{\varphi}^{-}\left(1+C_{3} N\right), \quad \Sigma_{\varphi} \approx \pm 1 \Longrightarrow \mathcal{R} \approx \pm \frac{C_{\varphi}^{-}}{\sqrt{6}}\left(1+C_{3} N\right), \\
& y-\frac{\Sigma_{\varphi}^{\prime}}{\Sigma_{\varphi}} \approx \frac{C_{3}}{1+C_{3} N} \quad \Longrightarrow \quad \zeta \approx \pm \frac{C_{\varphi}^{-}}{\sqrt{6}}\left(1+C_{3} N-\frac{1}{3} C_{3}\right) .
\end{aligned}
$$

For generic orbits $\left(C_{3} \neq 0\right)$ it follows that $\mathcal{R}$ and $\zeta$ diverge as $N \rightarrow-\infty$, while for orbits on the unstable manifold $\left(C_{3}=0\right)$ we obtain

$$
\lim _{N \rightarrow-\infty} \mathcal{R}=\lim _{N \rightarrow-\infty} \zeta= \pm \frac{C_{\varphi}^{-}}{\sqrt{6}} .
$$

We next consider orbits approaching the fixed points $\mathrm{A}_{\lambda}$ and $\mathrm{S}_{\lambda}$ for $0<\lambda<1 / \sqrt{3}$ into the future. Keeping only the future dominant terms we obtain for $A_{\lambda}$ :

$$
\begin{aligned}
\varphi_{c} \approx C_{\varphi}^{+}, \quad \Sigma_{\varphi} \approx \lambda & \Longrightarrow \quad \lim _{N \rightarrow \infty} \mathcal{R}=\frac{C_{\varphi}^{+}}{\sqrt{6} \lambda}, \\
y-\frac{\Sigma_{\varphi}^{\prime}}{\Sigma_{\varphi}} \approx 0 & \Longrightarrow \quad \lim _{N \rightarrow \infty} \zeta=\lim _{N \rightarrow \infty} \mathcal{R},
\end{aligned}
$$

and for $S_{\lambda}$ :

$$
\begin{aligned}
\varphi_{c} \approx C_{\varphi}^{+} e^{-3\left(1-\lambda^{2}\right) N}, \quad \Sigma_{\varphi} \approx \lambda & \Longrightarrow \quad \lim _{N \rightarrow \infty} \mathcal{R}=0, \\
y-\frac{\Sigma_{\varphi}^{\prime}}{\Sigma_{\varphi}} \approx-3\left(1-\lambda^{2}\right) & \Longrightarrow \quad \lim _{N \rightarrow \infty} \zeta=\left(2-\lambda^{2}\right) \lim _{N \rightarrow \infty} \mathcal{R}=0 .
\end{aligned}
$$

\footnotetext{
${ }^{26}$ See, for example, Uggla and Wainwright (2019) [15, equation (49), specialized to first order perturbations.

${ }^{27}$ The first equation is given as equation (2.11) in Uggla and Wainwright (2019) 16. The second equation follows from the perturbed Einstein equation $\psi_{\mathrm{v}}^{\prime}=c_{s}^{2} \boldsymbol{\delta}_{\mathrm{v}}+\Gamma$ (see equation (62a) in Uggla and Wainwright (2019) [27]) using the fact that $\Gamma=\left(1-c_{s}^{2}\right) \boldsymbol{\delta}_{\mathrm{v}}$ for a perturbed scalar field (see equation (3.3c) in Uggla and Wainwright (2019) [16.)

${ }^{28}$ Note that $\mathcal{R}^{\prime} / \mathcal{R}=\varphi_{\mathrm{c}}^{\prime} / \varphi_{\mathrm{c}}-\Sigma_{\varphi}^{\prime} / \Sigma_{\varphi}=y-\Sigma_{\varphi}^{\prime} / \Sigma_{\varphi}$.

${ }^{29}$ The first terms omitted have time dependence of the form $e^{2 N}$ and $e^{4 N}$.
} 
This establishes that the limits of $\mathcal{R}$ and $\zeta$ are equal and finite in both cases.

We next consider orbits approaching the fixed points $A_{\lambda}$ and $S_{\lambda}$ into the future, for the exceptional value $\lambda=0$. Keeping only the future dominant terms we obtain for $\mathrm{S}_{0}$ :

$$
\begin{aligned}
\varphi_{c} \approx C_{\varphi}^{+} e^{-3 N}, \quad \Sigma_{\varphi} \approx C_{1} e^{-3 N} & \Longrightarrow \quad \lim _{N \rightarrow \infty} \mathcal{R}=\frac{C_{\varphi}^{+}}{\sqrt{6} C_{1}}, \\
y-\frac{\Sigma_{\varphi}^{\prime}}{\Sigma_{\varphi}} \approx 0 & \Longrightarrow \quad \lim _{N \rightarrow \infty} \zeta=\lim _{N \rightarrow \infty} \mathcal{R},
\end{aligned}
$$

and for $\mathrm{A}_{0}$ :

$$
\begin{array}{ccc}
\varphi_{c} \approx C_{\varphi}^{+}, \quad \Sigma_{\varphi} \approx C_{1} e^{-3 N} & \Longrightarrow \quad \mathcal{R} \approx \frac{C_{\varphi}^{+}}{\sqrt{6} C_{1}} e^{3 N}, \\
1-\frac{1}{3}\left(y-\frac{\Sigma_{\varphi}^{\prime}}{\Sigma_{\varphi}}\right) \approx C_{2} e^{-2 N} \quad \Longrightarrow \quad \zeta \approx \frac{C_{\varphi}^{+} C_{2}}{3 \sqrt{6} C_{1}} e^{N} .
\end{array}
$$

This establishes that the limits of $\mathcal{R}$ and $\zeta$ are equal for the fixed point $\mathrm{S}_{0}$, while for orbits that approach the fixed point $\mathrm{A}_{0}, \mathcal{R}$ and $\zeta$ diverge as $N \rightarrow \infty$, but with $\mathcal{R}$ diverging faster than $\zeta$. In other words the conservation conjecture breaks down for generic orbits toward the future since the fixed point $\mathrm{A}_{0}$ is the future attractor, but it is valid for the restricted set of orbits that approach $\mathrm{S}_{0}$.

The latter result is related to those in [5] where it is shown that conservation of $\mathcal{R}$ and $\zeta$ for long wavelength perturbations breaks down in the case of ultra slow-roll inflation toward the future (see also [6, 7, 8, 9, 10, 11]). We can link our work to theirs by keeping additional terms in the expansion for $\varphi_{c}$ and $y{ }^{30}$ This leads to

$$
\begin{aligned}
\mathcal{R} & \approx \frac{C_{\varphi}^{+}}{3 \sqrt{6} C_{1}}\left[3 e^{3 N}\left(1+\frac{C_{2}}{2} e^{-2 N}\right)-C_{3}\right], \\
\zeta & \approx \frac{C_{\varphi}^{+}}{3 \sqrt{6} C_{1}}\left[e^{N} C_{2}-C_{3}\right] .
\end{aligned}
$$

These expansions are equivalent to equations (36) and (37) in [5], 31 which in [5] were derived using the usual heuristic approach to long wavelength perturbations ${ }^{32}$

Let us conclude this section by showing some of the global properties of $\mathcal{R}$ and $\zeta$ by numerically plotting some orbits in the state space and then illustrating the properties these solutions give rise to for $\mathcal{R}$ and $\zeta$. Since the ultra slow-roll case $\lambda=0$ yields generic unboundedness both toward the past and future, we restrict considerations to the case $0<\lambda<1 / \sqrt{3}$, which is represented by $\lambda=1 / \sqrt{6}$. We

\footnotetext{
${ }^{30}$ This yields $\varphi_{c} \approx C_{\varphi}^{+}\left(1-\frac{1}{3} e^{-3 N}+\frac{1}{2} e^{-2 N}\right), y \approx-C_{2} e^{-2 N}+C_{3} e^{-3 N}$.

${ }^{31}$ Note that $a^{3}=e^{3 N}$ and $k^{2} / \mathcal{H}^{2} \equiv Z \approx C_{2} e^{-2 N}$ as $N \rightarrow \infty$.

${ }^{32}$ The present dynamical systems approach can be used to obtain more refined approximations by making Picard expansions (see e.g. [28] and references therein), i.e., expansions based on the eigenvalues associated with the fixed points in the dynamical system. In, e.g., the case $\lambda=0$ and $\mathrm{A}_{0}$ this entails an expansion based on $e^{-2 N}$ and $e^{-3 N}$ where the equations for $\left(\Sigma_{\varphi}, Z, y\right)$ are solved for each coefficient of $e^{-2 N}, e^{-3 N}, e^{-4 N}, e^{-5 N}, e^{-6 N}, \ldots$, which when inserted into (71) leads to expansions in $C_{1}, C_{2} \propto k^{2}, C_{3}$ and since only $C_{2}$ involves $k^{2}$ correct approximate solutions do not involve series expansions in $k^{2}$ alone.
} 
then note that $\mathcal{R}$ and $\zeta$ become unbounded generically toward the past; it is only the unstable manifold of $\mathrm{M}_{ \pm}$that yield past boundedness of these quantities. For this reason we restrict our considerations to the orbits on the unstable manifold of $\mathrm{M}_{+}$, see Fig. 6. Note that for sufficiently small $Z_{\max }, \mathcal{R}$ and $\zeta$ are globally approximately conserved in this case.

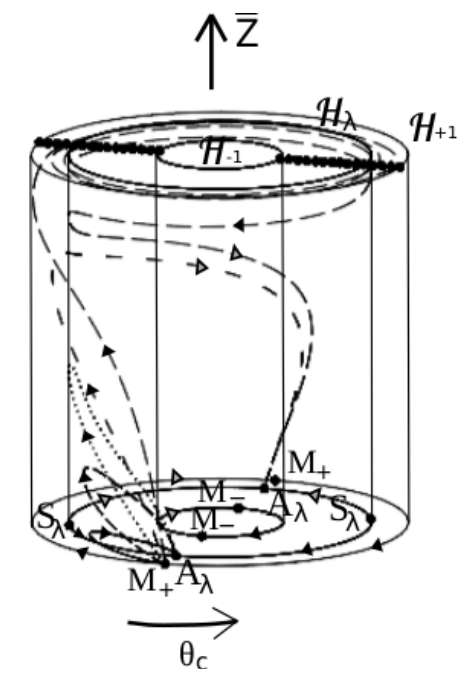

(a) State-space

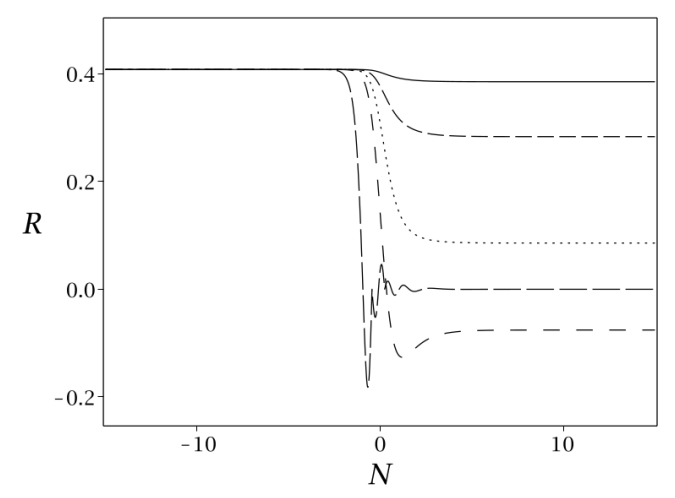

(c) $\mathcal{R}(N)$

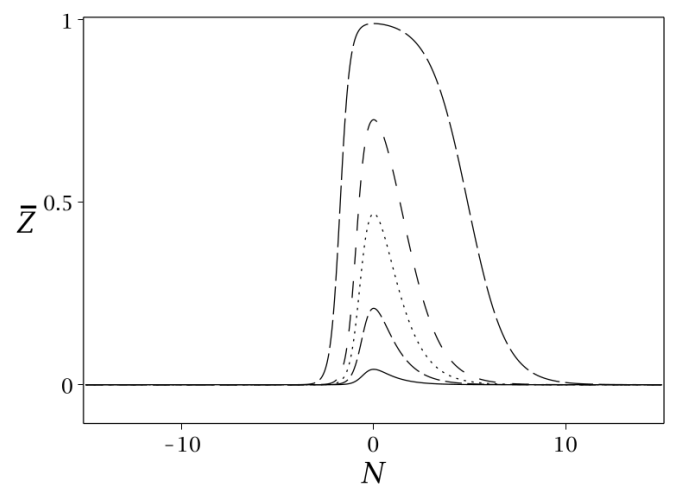

(b) $\bar{Z}(N)$

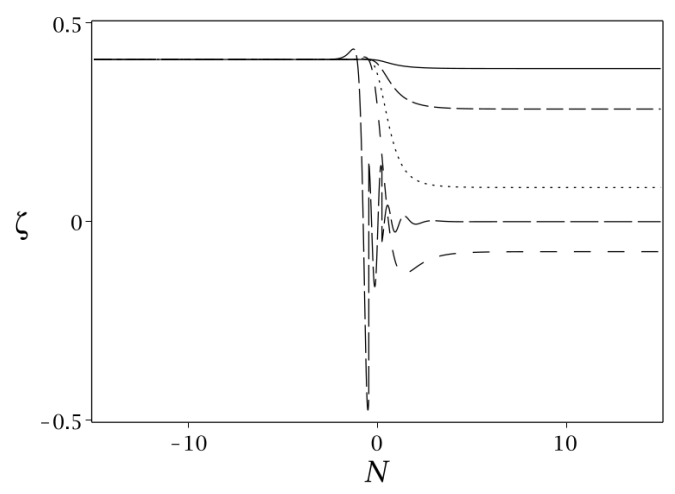

(d) $\zeta(N)$

Figure 6: Orbits on the unstable manifold of $\mathrm{M}_{+}$when $\lambda=1 / \sqrt{6}$, and the associated graphs for $\bar{Z}, \mathcal{R}$ and $\zeta$.

\section{Discussion}

We now indicate how our dynamical systems approach can be extended to apply to non-exponential scalar field potentials for which $\lambda(\varphi)$, defined by (9), is bounded ${ }^{33}$ Equation (12) describes the evolution of the background scalar field $\varphi$ through the first order differential equations for $\varphi$ and $\Sigma_{\varphi}$. For an exponential potential, we have seen that the equation for $\varphi$ decouples, but for a general potential one has to keep

\footnotetext{
${ }^{33}$ For how to globally deal with backgrounds with unbounded $\lambda(\varphi)$, see [29, 30, 31].
} 
both $\varphi$ and $\Sigma_{\varphi}$ as variables in the background state space $\mathcal{B}$. In order to obtain a compact background state space we introduce a new bounded scalar field variable

$$
\bar{\varphi}=f(\varphi), \quad-\infty<\varphi<\infty,
$$

where $f$ is a monotone increasing, differentiable and bounded function. We define $\bar{\varphi}_{ \pm}=\lim _{\varphi \rightarrow \pm \infty} f(\varphi)$, and also require that

$$
\lim _{\varphi \rightarrow \pm \infty}\left(\frac{d f}{d \varphi}\right)=0 .
$$

This results in coupled differential equations of the form

$$
\begin{aligned}
\bar{\varphi}^{\prime} & =\sqrt{6}\left(\frac{d \bar{\varphi}}{d \varphi}\right) \Sigma_{\varphi}, \\
\Sigma_{\varphi}^{\prime} & =-3\left(1-\Sigma_{\varphi}^{2}\right)\left(\Sigma_{\varphi}-\lambda\right),
\end{aligned}
$$

where $\lambda$ and $d \bar{\varphi} / d \varphi$ are expressed as (differentiable) functions of $\bar{\varphi}$ using the inverse $\varphi=f^{-1}(\bar{\varphi})$.

Thus the main change in generalizing from an exponential potential to a potential $V(\varphi)$ with bounded $\lambda(\varphi)$ is that a single differential equation for $\Sigma_{\varphi}$ is replaced by two coupled differential equations (81) for $\bar{\varphi}$ and $\Sigma_{\varphi}$, which means that the dimension of the background state space $\mathcal{B}$ increases from two to three (the third variable is $\bar{Z}$ ). The dimension of the perturbation state space $\mathcal{P}$ is unchanged at one: initially there are two perturbation variables but after compactification $\mathcal{P}$ is identified with a circle $S^{1}$. The perturbed KG equation, given by (16), has the same general structure, but the coefficients are now determined by the background quantities, $Z=k^{2} \mathcal{H}^{-2}, \Sigma_{\varphi}$, $\lambda(\varphi)$, and a new function $\Upsilon(\varphi)$, defined by

$$
\Upsilon(\varphi)=\frac{V_{, \varphi \varphi}}{6 V},
$$

which we also assume is bounded. In the case of an exponential potential $\Upsilon=\lambda^{2}$, and hence is constant.

If the scalars $\lambda$ and $\Upsilon$ are not constant but satisfy

$$
\lim _{\varphi \rightarrow \pm \infty} \lambda=\lambda_{ \pm}, \quad \lim _{\varphi \rightarrow \pm \infty} \Upsilon=\lambda_{ \pm}^{2},
$$

where $\lambda_{ \pm}=$const. we say that the potential $V(\varphi)$ is asymptotically exponential in the limit $\varphi \rightarrow \pm \infty$ and hence as $\bar{\varphi} \rightarrow \bar{\varphi}_{ \pm}$. For a such a potential the background state space $\mathcal{B}$ is defined by the inequalities $-1 \leq \Sigma_{\varphi} \leq 1, \bar{\varphi}_{-} \leq \bar{\varphi} \leq \bar{\varphi}_{+}, 0 \leq \bar{Z} \leq 1$. The boundary has six components given by $\Sigma_{\varphi}= \pm 1, \bar{\varphi}=\bar{\varphi}_{ \pm}, \overline{\bar{Z}}=0,1$, that are invariant sets. On account of 83 the dynamical system that is defined on the boundary components $\bar{\varphi}=\bar{\varphi}_{ \pm}$coincides with the dynamical system that governs a perturbed scalar field with exponential potential, with the parameter $\lambda$ given by $\lambda=\lambda_{ \pm}$. In this way the dynamical system developed in this paper for a scalar field with an exponential potential acts as a building block for dynamical systems that describe a scalar field with asymptotically exponential (or constant) potentials. 
The choice of the function $f(\varphi)$ in equation (79) depends on the form of the potential, and finding a suitable function involves some experimentation. We illustrate the process by considering a scalar field model introduced by Dimopoulos and Owen (2017) in [32, where the potential is given by

$$
V(\varphi)=V_{*}\left\{e^{\kappa \beta\left(1-\tanh \frac{\varphi}{\beta}\right)}-1\right\},
$$

depending on the constants $V_{*}, \kappa$ and $\beta$, with $\beta>0$ and $e^{2 \kappa \beta}>1^{34}$ This model describes so-called quintessential inflation ${ }^{35}$ in which the scalar field creates two phases of accelerated expansion, one at early times which leads to inflation and the other at late times which leads to quintessence. This potential is asymptotically exponential (even constant) since it can be verified that

$$
V(\varphi) \approx\left(e^{2 \kappa \beta}-1\right) V_{*}, \quad \varphi \rightarrow-\infty,
$$

which approximates an exponential potential with $\lambda_{-}=0$, and

$$
V(\varphi) \approx 2 \kappa \beta V_{*} e^{-2 \varphi / \beta}, \quad \varphi \rightarrow \infty
$$

which approximates an exponential potential with $\lambda_{+}=\sqrt{\frac{2}{3}} \beta^{-1}$. The potential thus has an inflationary plateau described by 85 and a quintessential tail described by (86), see figure 1 in 32 .

For this potential it is convenient to define the function $f(\varphi)$ in equation 790 by

$$
\bar{\varphi}=\tanh \frac{\varphi}{\beta},
$$

so that $\bar{\varphi}_{ \pm}=\lim _{\varphi \rightarrow \pm \infty} \bar{\varphi}= \pm 1$. It follows that

$$
\frac{d \bar{\varphi}}{d \varphi}=\beta^{-1}\left(1-\bar{\varphi}^{2}\right)
$$

as required in 81a), and one can verify that $\lambda(\bar{\varphi})$ and $\Upsilon(\bar{\varphi})$ are bounded and differentiable on $\bar{\varphi} \in[-1,1]$. Moreover, it can be verified that $\lambda(\bar{\varphi})$ and $\Upsilon(\bar{\varphi})$ satisfy (83), with $\lambda_{+}=\sqrt{\frac{2}{3}} \beta^{-1}$ and $\lambda_{-}=0$, which confirms that the potential (84) is asymptotically exponential (constant) when $\varphi \rightarrow+\infty(\varphi \rightarrow-\infty)$. The perturbation space for this potential will be treated in a forthcoming paper.

We conclude with some brief remarks on the structure of the state space $\mathcal{S}=\mathcal{B} \times \mathcal{P}$ for models with multiple sources. In a future paper we will generalize the analysis of a scalar field with exponential potential in this paper by adding dust (CDM) as a second source, with the two sources assumed to be non-interacting. The main change will be to add the density parameter for the dust, $\Omega_{m}$, as a second background matter variable. The background state space $\mathcal{B}$ will thus be three dimensional, with coordinates $\left(\Sigma_{\varphi}, \Omega_{m}, \bar{Z}\right)$, with $\bar{Z}$ describing the evolution of the background geometry represented by $\mathcal{H}$, as in the present paper.

${ }^{34} \beta=\sqrt{6 \alpha}$ in the notation used in [32].

${ }^{35}$ See Peebles and Vilenkin (1999) [33. 
There is, however, a significant increase in complexity as regards the perturbation space $\mathcal{P}$. In the present paper, one starts with two perturbation variables $\sqrt{36}$ but the compactified state space is the circle $S^{1}$. With two sources there will initially be four scalar perturbation variables (two for each component) and the process of compactification will lead to a three-sphere $S^{3}$ that is defined by a constraint equation. The perturbation space $\mathcal{P}$ will thus be three dimensional and compact, or four dimensional with one constraint.

\section{Acknowledgments}

AA is supported by CAMGSD, Instituto Superior Técnico by FCT/Portugal through UID/MAT/04459/2019 and UIDB/MAT/04459/2020 and project (GPSEinstein) PTDC/MAT-ANA/1275/2014. CU would like to thank the CAMGSD, Instituto Superior Técnico in Lisbon and the University of Waterloo, Canada, for kind hospitality.

\section{References}

[1] A. Alho, C. Uggla, and J. Wainwright. Perturbations of the lambda-cdm model in a dynamical systems perspective. JCAP, 09:045, 2019.

[2] D. H. Lyth and E. D. Stewart. The curvature perturbation in power law (e.g extended) inflation. Phys. Lett. B, 274:168, 1992.

[3] R. Durrer. The cosmic microwave background. Cambridge University Press, 2008.

[4] S. Weinberg. Cosmology. Oxford university press, 2008.

[5] A. E. Romano, S. Mooij, and M. Sasaki. Adiabaticity and gravity theory independent conservation laws for cosmological perturbations. Phys. Lett. B, 755:464, 2016.

[6] S. Mooij and G. A. Palma. Consistently violating the non-gaussian consistency relation. Journal of Cosmology and Astroparticle Physics, 11:025, 2015.

[7] N. C. Tsamis and R. P. Woodard. Improved estimates of cosmological perturbations. Phys. Rev. D, 69:084005, 2004.

[8] W. H. Kinney. Horizon crossing and inflation with large $\eta$. Phys. Rev. D, 72:023515, 2005.

[9] M. H. Namjoo, H. Firouzjahi, and M. Sasaki. Violation of non-gaussianity consistency relation in a single-field inflationary model. EPL (Europhysics Letters), 101:39001, 2013i.

\footnotetext{
${ }^{36}$ Initially there is in fact one perturbation variable $f$ that satisfies a second order differential equation. In order to derive a dynamical system we consider $f, f^{\prime}$ as two independent perturbation variables, which then define one angular variable $\theta$ using $f^{\prime}=f \tan \theta$.
} 
[10] X. Chen, H. Firouzjahi, M. H. Namjoo, and M. Sasaki. A single field inflation model withlarge local non-gaussianity. Europhys. Lett., 102(59001), 2013.

[11] J. Martin, H. Motohashi, and T. Suyama. Ultra slow-roll inflation and the non-gaussianity consistency relation. Phys. Rev. D, 87:023514, 2013.

[12] A. R. Liddle and D. H. Lyth. Cosmological inflation and large-scale structure. Cambridge University Press, 2000.

[13] J. Wainwright and G. F. R. Ellis. Dynamical systems in cosmology. Cambridge University Press, 1997.

[14] K. A. Malik and D. Wands. Cosmological perturbations. Physics Reports, 475:1-51, 2009.

[15] C. Uggla and J. Wainwright. Second order cosmological perturbations: simplified gauge change formulas. Class. Quantum Grav., 36:035004, 2019.

[16] C. Uggla and J. Wainwright. The general solution at large scale for second order perturbations in a scalar field dominated universe. Journal of Cosmology and Astroparticle Physics, 2019(06):021-021, 2019.

[17] J. Halliwell. Scalar fields in cosmology with an exponential potential. Phys. Lett. B, 185:341, 1987.

[18] A. A. Coley, J. Ibánez, and R. J. van den Hoogen. Homogeneous scalar field cosmologies with an exponential potential. Journal of Mathematical Physics, 38:17, 1997.

[19] E. J. Copeland, A. R. Liddle, and D. Wands. Exponential potentials and cosmological scaling solutions. Phys. Rev. D, 57:4686, 1998.

[20] L. A. Urena-Lopez. Unified description of the dynamics of quintessential scalar fields. JCAP, 2012:035, 2012.

[21] S. Tsujikawa. Quintessence: a review. Class. Quantum Grav., 30:214003, 2013.

[22] A. Alho and C. Uggla. Scalar field deformations of lambda-cdm cosmology. Phys. Rev. D, 92(10):103502, 2015.

[23] D. S. Salopek and J.R. Bond. Nonlinear evolution of long-wavelength metric fluctuations in inflationary models. Phys. Rev. D, 42:3936, 1990.

[24] I. Huston and K. A. Malik. Numerical calculation of second order perturbations. JCAP, 09:019, 2009.

[25] J. Martin and C.Ringeval. Inflation after wmap3: confronting the slow-roll and exact power spectra with cmb data. JCAP, 08:009, 2006.

[26] C. Uggla and J. Wainwright. Second-order cosmological perturbations: New conserved quantities and the general solution at super-horizon scale. Phys. Rev. D, 100:023544, 2019. 
[27] C. Uggla and J. Wainwright. Second order cosmological perturbations: dynamics. Phys. Rev. D, 98:103534, 2018.

[28] C. Uggla. Asymptotic cosmological solutions: orthogonal bianchi type-ii models. Class. Quantum Grav., 6(3):383, 1989.

[29] A. Alho, J. Hell, and C. Uggla. Global dynamics and asymptotics for monomial scalar field potentials and perfect fluids. Class. Quant. Grav., 32(14):145005, 2015.

[30] A. Alho and C. Uggla. Global dynamics and inflationary center manifold and slow-roll approximants. Journal of Mathematical Physics, 56(012502), 2015.

[31] A. Alho and C. Uggla. Inflationary $\alpha$-attractor cosmology: A global dynamical systems perspective. Phys. Rev. D, 95(8):083517, 2017.

[32] K. Dimopoulos and C. Owen. Quintessential inflation with $\alpha$-attractors. J. of Cosmology and Astroparticle Physics, 06:027, 2017.

[33] P. J. E. Peebles and A. Vilenkin. Quintessential inflation. Phys. Rev. D, 59:063505, 1999. 